Article

\title{
Phylogenetic Studies and Metabolite Analysis of Sticta Species from Colombia and Chile by Ultra-High Performance Liquid Chromatography-High Resolution-Q-Orbitrap-Mass Spectrometry
}

\author{
Laura Albornoz $^{1,+}$, Alfredo Torres-Benítez ${ }^{2,+} \mathbb{D}$, Miguel Moreno-Palacios ${ }^{3}$, Mario J. Simirgiotis ${ }^{2} \mathbb{D}$, \\ Saúl A. Montoya-Serrano ${ }^{4}$, Beatriz Sepulveda ${ }^{5}$, Elena Stashenko ${ }^{6}$ (D) Olimpo García-Beltrán $7,8, *(\mathbb{D})$ \\ and Carlos Areche $1, * \mathbb{D}$
}

\section{check for}

updates

Citation: Albornoz, L.

Torres-Benítez, A.; Moreno-Palacios,

M.; Simirgiotis, M.J.;

Montoya-Serrano, S.A.; Sepulveda,

B.; Stashenko, E.; García-Beltrán, O.;

Areche, C. Phylogenetic Studies and

Metabolite Analysis of Sticta Species

from Colombia and Chile by

Ultra-High Performance Liquid

Chromatography-High

Resolution-Q-Orbitrap-Mass

Spectrometry. Metabolites 2022, 12,

156. https://doi.org/10.3390/

metabo12020156

Academic Editor:

Hirokazu Kawagishi

Received: 18 January 2022

Accepted: 2 February 2022

Published: 8 February 2022

Publisher's Note: MDPI stays neutral with regard to jurisdictional claims in published maps and institutional affiliations.

Copyright: (C) 2022 by the authors. Licensee MDPI, Basel, Switzerland. This article is an open access article distributed under the terms and conditions of the Creative Commons Attribution (CC BY) license (https:// creativecommons.org/licenses/by/ $4.0 /)$.
1 Departamento de Química, Facultad de Ciencias, Universidad de Chile, Las Palmeras 3425, Nuñoa, Santiago 7800024, Chile; albornoznocachomail.c@hotmail.com

2 Instituto de Farmacia, Facultad de Ciencias, Universidad Austral de Chile, Campus Isla Teja, Valdivia 5090000, Chile; aljotobe19@hotmail.com (A.T.-B.); mario.simirgiotis@uach.cl (M.J.S.)

3 Laboratorio de Biología Evolutiva de Vertebrados, Departamento de Ciencias Biológicas, Universidad de Los Andes, Bogotá 111711, Colombia; mc.morenop@uniandes.edu.co

4 Laboratorio de Toxicología, Seccional Tolima, Instituto Nacional de Medicina Legal y Ciencias Forenses, Ibagué 730006, Colombia; samontoya@medicinalegal.gov.co

5 Departamento de Ciencias Químicas, Universidad Andres Bello, Campus Viña del Mar, Quillota 980, Viña del Mar 2520000, Chile; bsepulveda@uc.cl

6 Research Center of Excellence CENIVAM, CIBIMOL, Universidad Industrial de Santander, Building 45, UIS, Carrera 27, Calle 9, Bucaramanga 680002, Colombia; elena@tucan.uis.edu.co

7 Centro Integrativo de Biología y Química Aplicada (CIBQA), Universidad Bernardo O'Higgins, General Gana 1702, Santiago 8370854, Chile

8 Facultad de Ciencias Naturales y Matemáticas, Universidad de Ibagué, Carrera 22 Calle 67, Ibagué 730002, Colombia

* Correspondence: jose.garcia@unibague.edu.co (O.G.-B.); areche@uchile.cl (C.A.); Tel.: +57-8276-0010 (O.G.-B.); $+56-9883-77059$ (C.A.)

+ These authors contributed equally to this work.

\begin{abstract}
Eleven species of lichens of the genus Sticta, ten of which were collected in Colombia (S. pseudosylvatica S. luteocyphellata S. cf. andina S. cf. hypoglabra, S. cordillerana, S. cf. gyalocarpa S. leucoblepharis, S. parahumboldtii S. impressula, S. ocaniensis) and one collected in Chile (S. lineariloba), were analyzed for the first time using hyphenated liquid chromatography with high-resolution mass spectrometry. In the metabolomic analysis, a total of 189 peaks were tentatively detected; the analyses were divided in five (5) groups of compounds comprising lipids, small phenolic compounds, saturated acids, terpenes, and typical phenolic lichen compounds such as depsides, depsidones and anthraquinones. The metabolome profiles of these eleven species are important since some compounds were identified as chemical markers for the fast identification of Sticta lichens for the first time. Finally, the usefulness of chemical compounds in comparison to traditional morphological traits to the study of ancestor-descendant relationships in the genus was assessed. Chemical and morphological consensus trees were not consistent with each other and recovered different relationships between taxa.
\end{abstract}

Keywords: chemotaxonomyc; lichens; metabolomics; Sticta; phylogenetic; UHPLC-MS-MS

\section{Introduction}

Lichens constitute a mutualistic symbiosis with green algae and/or cyanobacteria [1], and in some cases present a tripartite symbiosis between different mycobionts and photobionts known as photosymbiodemas [2-4]. The genus Sticta (Schreb.) Ach. is the most species-diverse group of macrolichens in the family Lobariaceae, with about 120 species, and is characterized by a heteromeric thallus, with wide and rounded or elongated and 
truncated lobes, sometimes overlapping and rarely polyphilic, with smooth upper surface or with light ribs that may carry isidia or soralia, and the presence of cyphelas on the ventral surface of variable sizes dispersed in the tomentum [5-11]. In South America, the genus is found in Andean, sub-Andean, and moorland ecosystems, and develops in substrates of bark, soil, wood and rocks [5-12].

Chemical studies of the genus Sticta are scarce. In Sticta and other genera, most compounds are of fungal origin and their chemical variety is related to the environmental conditions in which the species develops $[13,14]$, which is observed in this work. From the earliest reports found for this genus, triterpenes were identified in the species $S$. billardierii, S. coronata, S. colensoi, and S. favicans $[15,16]$. The latest reports on species such as $S$. fuliginosa, $S$. weigelia, $S$. caulescens and $S$. santessonii show the presence of substances such as $7 \beta-$ acetoxy-22-hydroxy hopane usnic acid, ursolic acid, ergosterol peroxide and $\beta$-sitosterol [17]. The S. nylanderiana ethyl-3-formyl-2,4-dihydroxy-5,6-dimethylbenzoate, methyl-2,4-dihydroxy3,5,6-trimethylbenzoate, orsellinic acid, methyl orsellinate, ethyl orsellinate, lecanoric acid, 4-O-methyl gyrophoric acid and retigeric acid B compounds were isolated and identified [18]. In an unidentified species of the genus Sticta, the compounds stictamides A-C were isolated and identified. In addition, stictamide A acts as an inhibitor of the MMP12 protease (metallopeptidase 12) [19].

Currently, for the identification and elucidation of metabolites in complex extracts, the technique of ultra-high performance liquid chromatography-diode array detection (UHPLC-PDA) coupled to an electrospray ionization tandem mass spectrometer (ESI-MSMS) [20-26] is used. The Q-Exactive Focus is a hybrid high-resolution mass spectrometer used to detect and quantify small organic compounds via high-resolution accurate mass spectrometry. This machine combines UHPLC-PDA (ultrahigh pressure liquid chromatography with photodiode array) with an orbitrap, a quadrupole (Q) and a high-resolution collision cell (HRCD), which allows for high resolution diagnostic untargeted metabolomics and accurate determination of fragments [15-28]. This technique has been useful for the chemical study of several lichens of the genera Ramalina, Parmotrema and Usnea [23-30] conducted by our research group and others.

In this research, we analyzed the phytochemical profile of several species of the Sticta genus collected in different geographical regions of Colombia and Chile, based on UHPLC-DAD coupled with high-resolution electrospray ionization tandem mass spectrometry (ESI-MS-MS) for the first time. The eleven (11) lichens of the genus Sticta analyzed were S. pseudosylvatica S. luteocyphellata S. cf. andina S. cf. hypoglabra, S. cordillerana, S. cf. gyalocarpa S. leucoblepharis, S. parahumboldtii S. impressula, S. ocaniensis and S. Lineariloba (Figure 1). Based on the quantity and variety of chemical compounds found in the studied specimens, we complemented the chemical characterization with a maximum parsimony analysis based on the hypothesis that phytochemical compounds in lichens may show a phylogenetic signal, thus proving utility for chemotaxonomy.
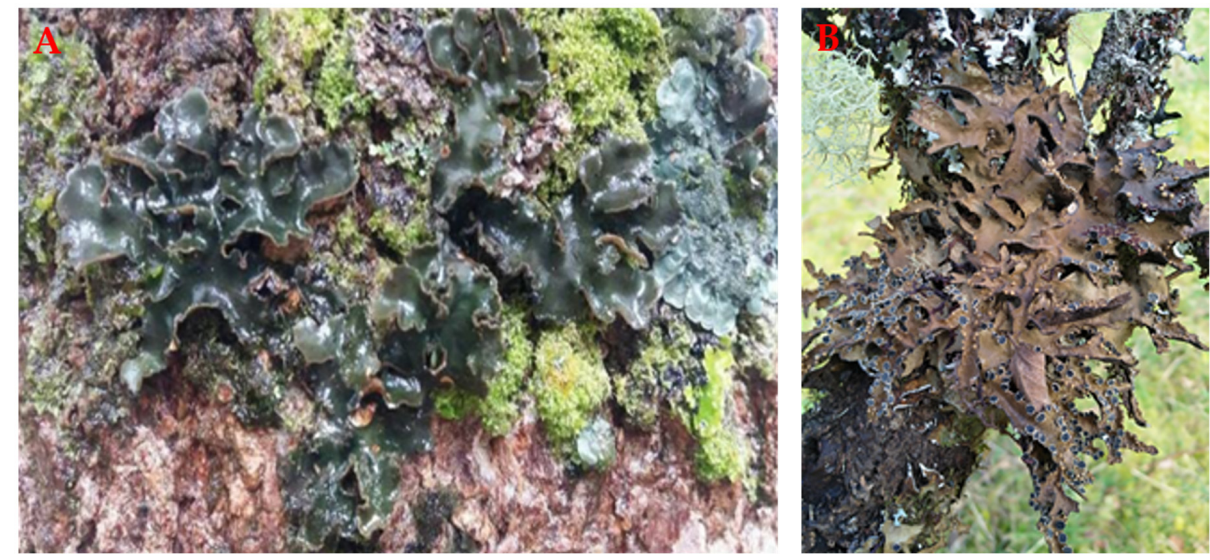

Figure 1. (A) S. pseudosylvatica (Colombia); (B) S. lineariloba (Chile). 


\section{Results and Discussion}

In this study, the metabolome profile of eleven species of Sticta lichens are reported for the first time. Lichen substances have gained considerable attention due to their potential health benefits and possible food nutraceutical or biotechnological applications [31]. Such compounds consist mostly of aliphatic and aromatic substances which proved to have biological and pharmacological activities compared to higher plants, in particular several depsidones and depsides which proved to be antioxidant, cytotoxic and anti-inflammatory agents, among several other bioactivities reported from the genus [31-35]. Their identification by hyphenated mass spectrometry liquid chromatography techniques is highly important for the fast metabolite profiling of these important biodiverse organisms, and the possibility of finding biomarkers that could be of help for their identification.

\subsection{Identification of Metabolites in 11 Lichen Species}

In this study, eleven species of the genus Sticta were analyzed and Figure 2 shows the chromatograms for the species S. pseudosylvatica and S. lineariloba. In the metabolomic analysis, a total of 189 peaks were detected, 41 of which were unknown compounds, and the analyses were divided in five groups of compounds as explained below (Table 1).
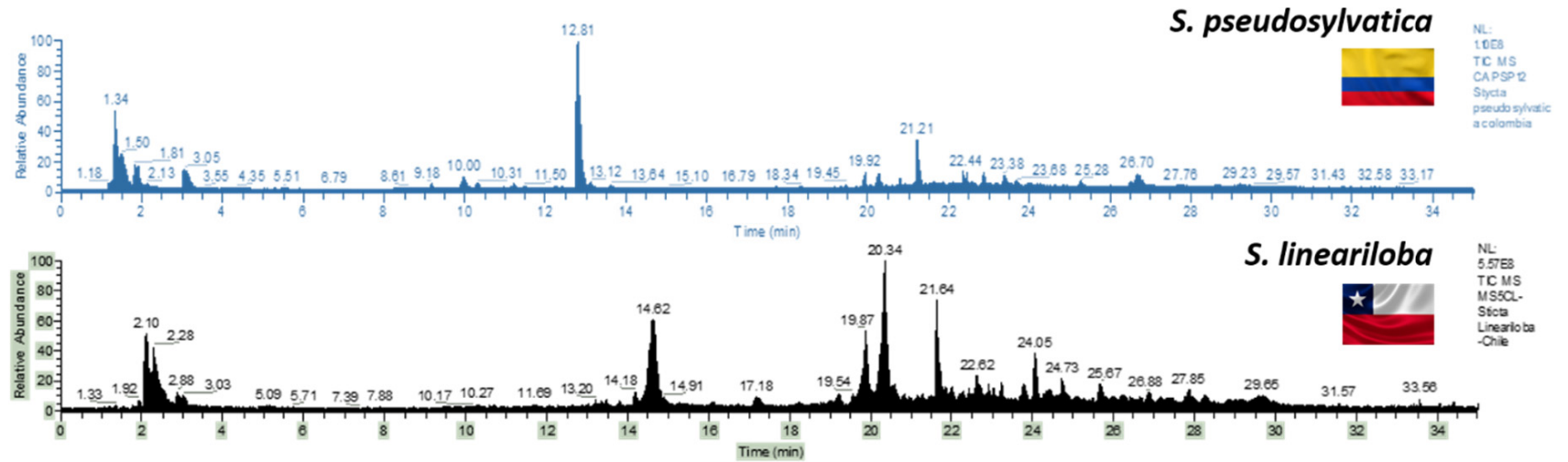

Figure 2. Chromatogram of the species S. pseudosylvatica and S. lineariloba.

Table 1. Identification of metabolites in 11 species of the genus Sticta from Colombia and Chile.

\begin{tabular}{|c|c|c|c|c|c|c|c|c|c|}
\hline Peak & Tentative Identification & [M-H]- & $\begin{array}{c}\text { Retention } \\
\text { Time (min) }\end{array}$ & $\begin{array}{l}\text { Theoretical } \\
\text { Mass }(\mathrm{m} / \mathrm{z})\end{array}$ & $\begin{array}{l}\text { Measured } \\
\text { Mass }(\mathrm{m} / \mathrm{z})\end{array}$ & $\begin{array}{c}\text { Accuracy } \\
(\mathrm{ppm})\end{array}$ & $\begin{array}{c}\text { Metabolite } \\
\text { Type }\end{array}$ & $\underset{(\mathrm{m} / \mathrm{z})}{\text { MS Ions }}$ & Lichen Species * \\
\hline $\begin{array}{l}1 \\
2\end{array}$ & $\begin{array}{l}\text { Gluconic Acid } \\
\text { Unknown }\end{array}$ & $\begin{array}{l}\mathrm{C}_{6} \mathrm{H}_{11} \mathrm{O}_{7} \\
\mathrm{C}_{11} \mathrm{H}_{5} \mathrm{O}_{5} \mathrm{~N}\end{array}$ & $\begin{array}{l}1.28 \\
1.28\end{array}$ & $\begin{array}{l}195.0509 \\
231.0184\end{array}$ & $\begin{array}{l}195.0505 \\
231.0195\end{array}$ & $\begin{array}{c}2.0 \\
-4.8\end{array}$ & Acid & 165.0401; & $\begin{array}{c}2 ; 3 ; 7 ; 9 ; 10 ; 11 \\
6\end{array}$ \\
\hline 3 & Manitol & $\mathrm{C}_{6} \mathrm{H}_{13} \mathrm{O}_{6}$ & 1.31 & 181.0712 & 181.0714 & -1.1 & Carbohidrate & $\begin{array}{l}\text { 181.0717; } \\
\text { 163.0606 }\end{array}$ & $7 ; 9 ; 10 ; 11$ \\
\hline 4 & Arabic acid & $\mathrm{C}_{5} \mathrm{H}_{9} \mathrm{O}_{6}$ & 1.29 & 165.0399 & 165.0401 & -1.2 & Acid & $\begin{array}{l}\text { 147.0293; } \\
\text { 113.0237; }\end{array}$ & 6 \\
\hline 5 & Citric Acid & $\mathrm{C}_{6} \mathrm{H}_{7} \mathrm{O}_{7}$ & 1.38 & 191.0196 & 191.0192 & 2.0 & Acid & $\begin{array}{l}129.0196 \\
111.0080\end{array}$ & $1 ; 2 ; 3 ; 4 ; 5 ; 6 ; 7 ; 8 ; 9 ; 11$ \\
\hline 6 & Unknown & $\mathrm{C}_{15} \mathrm{H}_{5} \mathrm{O}_{3} \mathrm{~N}_{2}$ & 1.38 & 261.0289 & 261.0300 & -4.2 & - & - & $1 ; 7 ; 8 ; 10$ \\
\hline 7 & 4-ethyl-2-Ethylisophthalic acid & $\mathrm{C}_{10} \mathrm{H}_{9} \mathrm{O}_{4}$ & 1.44 & 193.0504 & 193.0501 & 1.6 & A & 133.0288 & $1 ; 2 ; 3 ; 4 ; 6$ \\
\hline 8 & $\begin{array}{l}\text { Unknown } \\
\text { Unde deid }\end{array}$ & $\mathrm{C}_{8} \mathrm{H}_{10} \mathrm{O}_{5} \mathrm{~N}$ & $\begin{array}{l}1.44 \\
1.56\end{array}$ & 200.0563 & 200.0559 & $\begin{array}{l}1.0 \\
2.0\end{array}$ & - & 103.0200 & $\begin{array}{l}1 ; 2 ; 3 ; 4 ; 6 \\
1 ; 3 ; 4 ; 6\end{array}$ \\
\hline 9 & Unknown & $\mathrm{C}_{15} \mathrm{H}_{5} \mathrm{O}_{3} \mathrm{~N}_{2}$ & 1.64 & 261.0247 & 261.0278 & -11.8 & - & - & $\begin{array}{l}\text { 1, } \\
7 ; 8 ; 10\end{array}$ \\
\hline 10 & Isocitric Acid & $\mathrm{C}_{6} \mathrm{H}_{7} \mathrm{O}_{7}$ & 1.77 & 191.0195 & 191.0192 & 1.6 & Acid & 111.0079; & $1 ; 2 ; 3 ; 4 ; 6 ; 7$ \\
\hline 11 & Unknown & $\mathrm{C}_{7} \mathrm{H}_{9} \mathrm{O}_{7}$ & 1.91 & 205.0352 & 205.0348 & 2.0 & - & $\begin{array}{l}\text { 187.0245; } \\
\text { 173.0087; } \\
\text { 121.1131 }\end{array}$ & 2 \\
\hline 12 & Unknown & $\mathrm{C}_{7} \mathrm{H}_{7} \mathrm{O}_{6}$ & 2.24 & 187.0246 & 187.0243 & 1.6 & - & $\begin{array}{l}\text { 125.0237; } \\
\text { 137.2503; }\end{array}$ & 2 \\
\hline 13 & 2-Ethylisophthalic acid & $\mathrm{C}_{10} \mathrm{H}_{9} \mathrm{O}_{4}$ & 2.85 & 193.0505 & 193.0501 & 2.0 & $\mathrm{~A}$ & 161.0240; & $1 ; 2 ; 3 ; 4 ; 6 ; 7 ; 8 ; 9 ; 10$ \\
\hline $\begin{array}{l}14 \\
15 \\
16 \\
17 \\
18 \\
19 \\
20\end{array}$ & $\begin{array}{l}\text { Trihydroxy benzaledehyde } \\
\text { 2-Hydroxyisophthalic acid } \\
\text { 2,4-dihydroxy benzaldehyde } \\
\text { 4-O-Demethylglomellic acid } \\
\text { Unknow } \\
\text { Unknow } \\
\text { Grayanic acid }\end{array}$ & $\begin{array}{l}\mathrm{C}_{7} \mathrm{H}_{5} \mathrm{O}_{4} \\
\mathrm{C}_{8} \mathrm{H}_{5} \mathrm{O}_{5} \\
\mathrm{C}_{7} \mathrm{H}_{5} \mathrm{O}_{3} \\
\mathrm{C}_{24} \mathrm{H}_{25} \mathrm{O}_{9} \\
\mathrm{C}_{22} \mathrm{H}_{23} \mathrm{O}_{7} \\
\mathrm{C}_{7} \mathrm{H}_{11} \mathrm{O}_{5} \\
\mathrm{C}_{23} \mathrm{H}_{25} \mathrm{O}_{7}\end{array}$ & $\begin{array}{l}4.81 \\
7.63 \\
8.02 \\
8.65 \\
8.77 \\
8.86 \\
8.91\end{array}$ & $\begin{array}{l}153.0188 \\
181.0137 \\
137.0238 \\
457.1476 \\
399.1444 \\
175.0606 \\
413.1600\end{array}$ & $\begin{array}{l}153.0189 \\
181.0141 \\
137.0239 \\
457.1499 \\
399.1413 \\
175.0611 \\
413.1569\end{array}$ & $\begin{array}{c}-0.7 \\
-2.2 \\
-0.7 \\
-5.0 \\
7.8 \\
-2.9 \\
7.5\end{array}$ & $\begin{array}{l}\text { A } \\
\text { A } \\
\text { A } \\
\text { d } \\
- \\
- \\
\text { d }\end{array}$ & $\begin{array}{c}133.0290 \\
137.0238 \\
121.0288 \\
- \\
- \\
181.06503\end{array}$ & $\begin{array}{c}1 ; 2 ; 3 ; 4 \\
4 \\
1 ; 2 ; 3 ; 4 \\
5 ; 8 \\
8 \\
5 \\
8\end{array}$ \\
\hline 21 & Unknown & $\mathrm{C}_{13} \mathrm{H}_{16} \mathrm{O}_{7} \mathrm{~N}$ & 8.98 & 298.0940 & 298.0927 & 4.4 & - & $\begin{array}{l}\text { 175.0609; } \\
\text { 283.0210; } \\
202.0696 ; \\
\text { 137.0603; }\end{array}$ & 5 \\
\hline 22 & Unknown & $\mathrm{C}_{11} \mathrm{H}_{9} \mathrm{O}_{7}$ & 9.33 & 253.0361 & 253.0348 & 5.1 & - & $\begin{array}{l}\text { 181,0505; } \\
249.3808 \\
173.4203\end{array}$ & 5 \\
\hline 23 & Atranol & $\mathrm{C}_{8} \mathrm{H}_{7} \mathrm{O}_{3}$ & 9.44 & 151.0395 & 151.0396 & -0.7 & A & $\begin{array}{l}\text { 123.0445; } \\
135.0445\end{array}$ & 3 \\
\hline 24 & Unknown & $\mathrm{C}_{24} \mathrm{O}_{23}$ & 9.48 & 439.1371 & 439.1393 & -5.0 & - & $\begin{array}{l}\text { 151.0397; } \\
\text { 105.0948; } \\
\text { 121.4871; }\end{array}$ & 5 \\
\hline
\end{tabular}


Table 1. Cont.

\begin{tabular}{|c|c|c|c|c|c|c|c|c|c|}
\hline Peak & Tentative Identification & {$[\mathrm{M}-\mathrm{H}]-$} & $\begin{array}{l}\text { Retention } \\
\text { Time (min) }\end{array}$ & $\begin{array}{l}\text { Theoretical } \\
\text { Mass }(m / z)\end{array}$ & $\begin{array}{l}\text { Measured } \\
\text { Mass }(m / z)\end{array}$ & $\begin{array}{l}\text { Accuracy } \\
(\mathrm{ppm})\end{array}$ & $\begin{array}{l}\text { Metabolite } \\
\text { Type } * *\end{array}$ & MS Ions $(m / z)$ & Lichen Species* \\
\hline 25 & 5,7-Dihydroxy-6-methylphthalide & $\mathrm{C}_{9} \mathrm{H}_{7} \mathrm{O}_{4}$ & 9.64 & 179.0344 & 179.0347 & -1.6 & A & $\begin{array}{l}\text { 135.0444; } \\
107.0494\end{array}$ & $4 ; 5 ; 7 ; 8 ; 10$ \\
\hline 26 & Unknown & $\mathrm{C}_{16} \mathrm{H}_{15} \mathrm{O}_{10}$ & 9.73 & 367.0665 & 367.0639 & 7.1 & - & $\stackrel{107.0494}{-}$ & $1 ; 4$ \\
\hline 27 & Unknown & $\mathrm{C}_{30} \mathrm{H}_{27} \mathrm{O}_{13} \mathrm{~N}$ & 10.03 & 609.1475 & 609.1482 & -1.1 & - & - & $1 ; 4 ; 6$ \\
\hline 28 & Unknow & $\begin{array}{l}\mathrm{C} 18 \mathrm{H} 15 \mathrm{O} 4 \\
\mathrm{C}\end{array}$ & 10.31 & 295.0970 & 295.0935 & 11.8 & - & 二 & $\begin{array}{l}1 ; 4 ; 6 \\
9\end{array}$ \\
\hline 29 & Unknow & $\mathrm{C}_{17} \mathrm{H}_{13} \mathrm{O}_{6}$ & 11.31 & 313.0712 & 313.0724 & -3.8 & - & - & $1 ; 2 ; 3 ; 4 ; 5$ \\
\hline 30 & 1,5-Pentanedicarboxylic acid & $\mathrm{C}_{7} \mathrm{H}_{11} \mathrm{O}_{4}$ & 10.56 & 159.0657 & 159.0660 & -1.9 & $\mathrm{~L}$ & 115.0758 & 5 \\
\hline 31 & Unknown & $\mathrm{C}_{29} \mathrm{H}_{25} \mathrm{O}_{13} \mathrm{~N}$ & 10.54 & 595.1316 & 595.1326 & -1.7 & - & - & 3 \\
\hline 32 & Didechlorolecideoidin & $\mathrm{C}_{17} \mathrm{H}_{13} \mathrm{O}_{7}$ & 10.64 & 329.0661 & 329.0676 & -4.6 & $\mathrm{D}$ & $\begin{array}{c}209.0456 ; \\
285.0776 ; \\
151.0396 ; \\
179.0347 ; 123.0443\end{array}$ & 3 \\
\hline 33 & Decahydroxyoxopentacosanoic acid & $\mathrm{C}_{25} \mathrm{H}_{47} \mathrm{O}_{13}$ & 10.71 & 555.3017 & 555.3047 & -5.4 & $\mathrm{~L}$ & 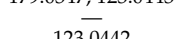 & 8 \\
\hline $\begin{array}{l}34 \\
35\end{array}$ & $\begin{array}{l}\text { Orsellinic acid } \\
\text { Unknow }\end{array}$ & $\begin{array}{c}\mathrm{C}_{8} \mathrm{H}_{7} \mathrm{O}_{4} \\
\mathrm{C}_{21} \mathrm{H}_{19} \mathrm{O}_{12}\end{array}$ & $\begin{array}{l}11.04 \\
11.04\end{array}$ & $\begin{array}{l}167.0347 \\
463.0877\end{array}$ & $\begin{array}{l}167.0344 \\
463.0893\end{array}$ & $\begin{array}{c}1.8 \\
-3.4\end{array}$ & A & 123.0442 & $2 ; 3 ; 4 ; 5 ; 6$ \\
\hline $\begin{array}{l}35 \\
36\end{array}$ & $\begin{array}{l}\text { Unknow } \\
\text { Unknow }\end{array}$ & $\begin{array}{l}\mathrm{C}_{21} \mathrm{H}_{19} \mathrm{U}_{12} \\
\mathrm{C}_{10} \mathrm{H}_{9} \mathrm{O}_{5}\end{array}$ & $\begin{array}{l}11.04 \\
11.07\end{array}$ & $\begin{array}{l}463.08 / 7 \\
209.0450\end{array}$ & $\begin{array}{l}463.0893 \\
209.0458\end{array}$ & $\begin{array}{l}-3.4 \\
-3.8\end{array}$ & - & - & $\begin{array}{c}3 \\
5 ; 6\end{array}$ \\
\hline 37 & Nor $8^{\prime}$-methylconstictic acid & $\mathrm{C}_{21} \mathrm{H}_{19} \mathrm{O}_{11}$ & 11.11 & 447.0927 & 447.0942 & -3.4 & $\mathrm{~d}$ & 209.0455 & 2 \\
\hline 38 & Unknow & $\mathrm{C}_{17} \mathrm{H}_{13} \mathrm{O}_{6}$ & 11.20 & 313.0712 & 313.0720 & -2.5 & - & - & 7 \\
\hline 39 & Metil-2,6-dihidroxibenzoate & $\mathrm{C}_{8} \mathrm{H}_{7} \mathrm{O}_{4}$ & 11.21 & 167.0344 & 167.0346 & -1.1 & A & $\begin{array}{l}109.0287 \\
137.0238 \\
195.0665\end{array}$ & 9 \\
\hline 40 & Hypostictic acid isomer & $\mathrm{C}_{19} \mathrm{H}_{15} \mathrm{O}_{8}$ & 11.36 & 371.0778 & 371.0782 & -1.1 & $\mathrm{D}$ & $\begin{array}{l}\text { 19.0665; } \\
\text { 327.0885; } \\
341.0679 ; \\
179.0347\end{array}$ & $1 ; 3 ; 4 ; 7$ \\
\hline 41 & Unknow & $\mathrm{C}_{19} \mathrm{H}_{16} \mathrm{O}_{9}$ & 11.69 & 402.0825 & 402.0841 & -3.98 & - & - & 11 \\
\hline 42 & Fumarprotocetraric acid derivative & $\mathrm{C}_{17} \mathrm{H}_{11} \mathrm{O}_{6}$ & 11,70 & 311.0556 & 311.0564 & -2.5 & $\mathrm{~d}$ & - & $7 ; 10$ \\
\hline 43 & 4,5-Dihydroxy-2-nonenoic acid & $\mathrm{C}_{9} \mathrm{H}_{15} \mathrm{O}_{4}$ & 12.11 & 187.0974 & 187.0977 & -1.6 & $\mathrm{~L}$ & $\begin{array}{l}\text { 171.1025; } \\
143.1072\end{array}$ & $1 ; 2 ; 3 ; 4 ; 9 ; 10$ \\
\hline 44 & 2,4-Dicarboxy-3-hydroxy-5- & $\mathrm{C}_{10} \mathrm{H}_{9} \mathrm{O}_{6}$ & 12.14 & 225.0407 & 225.0399 & 3.5 & A & $\begin{array}{c}\text { 181.0503; } \\
167.0346 \cdot 149.0240\end{array}$ & 4 \\
\hline 45 & $\begin{array}{l}\text { methoxytowene } \\
\text { Unknown }\end{array}$ & $\mathrm{C}_{17} \mathrm{H}_{13} \mathrm{O}_{6}$ & 12.16 & 313.0724 & 313.0727 & -1.0 & - & $\begin{array}{c}107.0040 ; 149.0240 \\
-\end{array}$ & 4 \\
\hline 46 & Unknown & $\mathrm{C}_{18} \mathrm{H}_{15} \mathrm{O}_{7}$ & 12.37 & 343.0818 & 343.0826 & -2.3 & - & - & $7 ; 9$ \\
\hline 47 & Unknown & $\mathrm{C}_{21} \mathrm{H}_{17} \mathrm{O}_{12}$ & 12.40 & 461.0737 & 461.0720 & 3.7 & - & - & 6 \\
\hline 48 & Unknown & $\mathrm{C}_{20} \mathrm{H}_{17} \mathrm{O}_{8}$ & 12.52 & 385.0939 & 385.0923 & 4.1 & - & 二 & $1: 2 ; 3$ \\
\hline $\begin{array}{l}48 \\
49\end{array}$ & Unknow & $\mathrm{C}_{10} \mathrm{H}_{9} \mathrm{O}_{4}^{8}$ & 12.81 & 193.0501 & 193.0502 & $\begin{array}{c}4.1 \\
-0.5\end{array}$ & - & 二 & $1 ; 2 ; 3 ; 4 ; 5 ; 6 ; 7 ; 8 ; 9 ; 10$ \\
\hline 50 & Unknow & $\mathrm{C}_{27} \mathrm{H}_{45} \mathrm{O}_{6}$ & 13.04 & 465.3216 & 465.3231 & -3.2 & - & - & $1,2,0,4,0,0,1,0,10$ \\
\hline 51 & 2,4-dihydroxy benzaldehyde & $\mathrm{C}_{7} \mathrm{H}_{5} \mathrm{O}_{3}$ & 13.07 & 137.0237 & 137.0239 & -1.5 & $\mathrm{~A}$ & $\begin{array}{l}\text { 121.0288 } \\
\text { 373.0573; }\end{array}$ & 5 \\
\hline 52 & Consalizinic acid derivative I & $\mathrm{C}_{19} \mathrm{H}_{13} \mathrm{O}_{11}$ & 13.36 & 417.0458 & 417.0474 & -3.8 & $\mathrm{D}$ & $\begin{array}{l}387.0373 \\
225.0406, \\
177.0193\end{array}$ & 11 \\
\hline 53 & $\begin{array}{l}\text { 4-Ethoxy-3-formyl-2-hydroxy-6- } \\
\text { methylbenzoic acid }\end{array}$ & $\mathrm{C}_{11} \mathrm{H}_{11} \mathrm{O}_{5}$ & 13.41 & 223.0614 & 223.0606 & 3.6 & A & $\begin{array}{l}\text { 177.0190; } \\
\text { 133.0296; }\end{array}$ & $1 ; 2 ; 3 ; 4 ; 7 ; 9 ; 10$ \\
\hline 54 & $\begin{array}{l}\text { metnyibenzoic acia } \\
\text { Unknow }\end{array}$ & $\mathrm{C}_{20} \mathrm{H}_{15} \mathrm{O}_{8}$ & 13.47 & 383.0767 & 383.0781 & -3.6 & - & 103.0290 & 1 \\
\hline 55 & Consalizinic acid derivative II & $\mathrm{C}_{20} \mathrm{H}_{17} \mathrm{O}_{11}$ & 13,47 & 433.0771 & 433.0787 & -3.7 & $\mathrm{D}$ & $\begin{array}{l}401.0524 ; \\
417.0474 ; \\
373.0574\end{array}$ & 11 \\
\hline 56 & Cynodontin or Citreorosein isomer, & $\mathrm{C}_{15} \mathrm{H}_{9} \mathrm{O}_{6}$ & 13.71 & 285.0399 & 285.0410 & -3.9 & Anthraquinone & $\begin{array}{l}151.0396 \\
137.0237 \\
3730573\end{array}$ & 2 \\
\hline 57 & Consalizinic acid derivative I isomer & $\mathrm{C}_{19} \mathrm{H}_{13} \mathrm{O}_{11}$ & 13.78 & 417.0458 & 417.0474 & -3.84 & $\mathrm{D}$ & $\begin{array}{c}\text { 343.0467,77.0190; } \\
401.0523\end{array}$ & 10 \\
\hline 58 & Unknow & $\mathrm{C}_{30} \mathrm{H}_{47} \mathrm{O}_{7}$ & 13.79 & 519.3322 & 519.3337 & -2.9 & - & - & 3 \\
\hline 59 & 1,4,5,6,8-Pentahydroxy-3- & $\mathrm{C}_{15} \mathrm{H}_{9} \mathrm{O}_{7}$ & 13.87 & 301.0348 & 301.0361 & -4.3 & Anthraquinone & - & 4 \\
\hline $\begin{array}{l}60 \\
61\end{array}$ & $\begin{array}{c}\text { ethylanthraquinone } \\
\text { Unknow } \\
\text { Unknow }\end{array}$ & $\begin{array}{l}\mathrm{C}_{19} \mathrm{H}_{15} \mathrm{O}_{4} \\
\mathrm{C}_{14} \mathrm{H}_{13} \mathrm{O}_{7}\end{array}$ & $\begin{array}{l}13.87 \\
13.99\end{array}$ & $\begin{array}{l}307.0970 \\
293.0661\end{array}$ & $\begin{array}{l}307.0939 \\
293.0674\end{array}$ & $\begin{array}{l}10.0 \\
-4.4\end{array}$ & - & - & $\begin{array}{l}8 \\
3\end{array}$ \\
\hline 62 & Haemathamnolic acid isomer & $\mathrm{C}_{19} \mathrm{H}_{15} \mathrm{O}_{10}$ & 14.18 & 403.0665 & 403.0681 & -3.97 & $\mathrm{D}$ & $\begin{array}{l}359.0781 \\
371.0414 \\
209.0455\end{array}$ & 11 \\
\hline 63 & Fumarprotocetraric acid derivative & $\mathrm{C}_{17} \mathrm{H}_{11} \mathrm{O}_{6}$ & 14.78 & 311.0567 & 311.0569 & -0.6 & d & $\overline{3570625}$ & $1 ; 2 ; 3 ; 7 ; 10$ \\
\hline 64 & Constictic acid & $\mathrm{C}_{19} \mathrm{H}_{13} \mathrm{O}_{10}$ & 14.61 & 401.0509 & 401.0528 & -4.74 & $\mathrm{D}$ & $\begin{array}{l}357.0625 ; \\
313.0726 ; \\
283.0619 ; \\
255.0670 ; \\
121.0289\end{array}$ & 11 \\
\hline 65 & Hypostictic acid isomer & $\mathrm{C}_{19} \mathrm{H}_{15} \mathrm{O}_{8}$ & 14.98 & 371.0781 & 371.0767 & 3.8 & $\mathrm{D}$ & $\begin{array}{c}\text { 327.0289; } \\
195.0664 \cdot 179,0347\end{array}$ & 5 \\
\hline 66 & Thelephoric acid & $\mathrm{C}_{18} \mathrm{H}_{7} \mathrm{O}_{8}$ & 14.81 & 351.0141 & 351.0154 & -3.7 & Terphenylquinones & 5 & 3 \\
\hline 67 & Methylstictic acid & $\mathrm{C}_{20} \mathrm{H}_{15} \mathrm{O}_{9}$ & 15.26 & 399.0716 & 399.0728 & -3.0 & $\mathrm{D}$ & $\begin{array}{l}371.0779 \\
193.0504\end{array}$ & $2 ; 7$ \\
\hline 68 & Nor $8^{\prime}$-metilconstictic acid & $\mathrm{C}_{21} \mathrm{H}_{19} \mathrm{O}_{11}$ & 15.28 & 447.0927 & 447.0944 & -3.80 & $\mathrm{D}$ & $\begin{array}{l}\text { 401.0524; } \\
209.0455 \\
355.0468 \\
329.0674\end{array}$ & 11 \\
\hline 69 & Protocetraric acid & $\mathrm{C}_{18} \mathrm{H}_{13} \mathrm{O}_{9}$ & 17.17 & 373.0560 & 373.0574 & -3.75 & $\mathrm{D}$ & $\begin{array}{l}\text { 31.0568; } \\
227.0352 ; \\
267.0669 ; \\
285.0777 \\
267.0673 ;\end{array}$ & 11 \\
\hline 70 & Hypoconstictic acid & $\mathrm{C}_{19} \mathrm{H}_{15} \mathrm{O}_{9}$ & 17.27 & 387.0716 & 387.0729 & -3.4 & $\mathrm{D}$ & $\begin{array}{c}311.0552 ; \\
149.0238 \\
343.0827 .167 .0345\end{array}$ & 3 \\
\hline 71 & Unknow & $\mathrm{C}_{14} \mathrm{H}_{13} \mathrm{O}_{6}$ & 17.84 & 277.0712 & 277.0724 & -4.3 & - & $\begin{array}{c}343.0827,107.0345 \\
-\end{array}$ & $3 ; 6$ \\
\hline 72 & 12,13,15-Trihydroxy-9-octadecenoic acid & $\mathrm{C}_{18} \mathrm{H}_{15} \mathrm{O}_{5}^{6}$ & 18.11 & 329.2328 & 329.2340 & -3.6 & $\mathrm{~L}$ & 285.1716; & $2 ; 6$ \\
\hline 73 & Unknow & $\mathrm{C}_{20} \mathrm{H}_{13} \mathrm{O}_{8}$ & 18.19 & 381.0610 & 381.0626 & -4.2 & - & $\begin{array}{l}-\overline{-} \\
343.0468 \\
269.0458\end{array}$ & $3 ; 4$ \\
\hline 74 & Salazinic acid & $\mathrm{C}_{18} \mathrm{H}_{11} \mathrm{O}_{10}$ & 18.21 & 387.0352 & 387.0368 & -4.13 & $\mathrm{D}$ & $\begin{array}{l}241.0507 ; \\
325.0365 ; \\
299.0569\end{array}$ & 11 \\
\hline 75 & Unknown & $\mathrm{C}_{10} \mathrm{H}_{9} \mathrm{O}_{4}$ & 18.45 & 193.0505 & 193.0501 & 2.1 & - & 299.000 & $3 ; 4$ \\
\hline 76 & Unknown & $\mathrm{C}_{30} \mathrm{H}_{47} \mathrm{O}_{7}$ & 18.61 & 519.3322 & 519.3319 & 0.6 & - & - & 3 \\
\hline 77 & Menegazziaic acid & $\mathrm{C}_{18} \mathrm{H}_{13} \mathrm{O}_{9}$ & 18.79 & 373.0560 & 373.0575 & -4.0 & $\mathrm{D}$ & $\begin{array}{l}311,0570 ; \\
255.0666 ; \\
329.0679 \\
327.0526\end{array}$ & 3 \\
\hline 78 & Norstictic acid & $\mathrm{C}_{18} \mathrm{H}_{11} \mathrm{O}_{9}$ & 18.86 & 371.0403 & 371.0417 & -3.8 & $\mathrm{D}$ & 151.0396; & 3 \\
\hline 79 & Unknow & $\mathrm{C}_{22} \mathrm{H}_{19} \mathrm{O}_{10}$ & 18.88 & 443.0978 & 443.0996 & -4.1 & - & $\begin{array}{l}123.0444 ; \\
359.0417 \\
315.0520\end{array}$ & 3 \\
\hline 80 & Physodalic acid & $\mathrm{C}_{20} \mathrm{H}_{15} \mathrm{O}_{10}$ & 18.99 & 415.0665 & 415.0681 & -3.85 & $\mathrm{D}$ & $\begin{array}{l}343.0832 ; \\
387,0367 ; \\
373.0573 ; \\
401.0525\end{array}$ & 11 \\
\hline
\end{tabular}


Table 1. Cont.

\begin{tabular}{|c|c|c|c|c|c|c|c|c|c|}
\hline Peak & Tentative Identification & {$[\mathrm{M}-\mathrm{H}]-$} & $\begin{array}{l}\text { Retention } \\
\text { Time (min) }\end{array}$ & $\begin{array}{l}\text { Theoretical } \\
\text { Mass }(m / z)\end{array}$ & $\begin{array}{l}\text { Measured } \\
\text { Mass }(m / z)\end{array}$ & $\underset{(\mathrm{ppm})}{\text { Accuracy }}$ & $\underset{* *}{\operatorname{Metabolite~Type}}$ & MS Ions $(m / z)$ & Lichen Species * \\
\hline 81 & Unknow & $\mathrm{C}_{26} \mathrm{H}_{19} \mathrm{O}_{10}$ & 19.00 & 491.0978 & 491.0997 & -3.9 & - & - & 3 \\
\hline 82 & $\begin{array}{l}\text { Derivative methyl } \\
\text { 8-hydroxy-4-0-demethylbarbatate }\end{array}$ & $\mathrm{C}_{19} \mathrm{H}_{19} \mathrm{O}_{9}$ & 19.05 & 391.1045 & 391.1029 & 4.1 & d & 359.0788 & 3 \\
\hline 83 & $12,13,15$-Trihydroxy-9-octadecenoic acid & $\mathrm{C}_{18} \mathrm{H}_{33} \mathrm{O}_{5}$ & 19.05 & 329.2328 & 329.2336 & -2.4299 & $\mathrm{~L}$ & $-\overline{-}$ & 7 \\
\hline 84 & Haemoventosin & $\mathrm{C}_{15} \mathrm{H}_{11} \mathrm{O}_{7}$ & 19.16 & 303,0519 & 303.0505 & 4.7 & Naphthaquinone & $\begin{array}{l}259.0619 ; \\
231.0667 \\
189.0560 ; \\
415.0680 \\
371.0780\end{array}$ & 3 \\
\hline 85 & $\alpha$-acetilconstictic acid derivative I & $\mathrm{C}_{21} \mathrm{H}_{17} \mathrm{O}_{11}$ & 19.22 & 445.0771 & 445.0786 & -3.3 & $\mathrm{D}$ & $\begin{array}{l}\text { 427.0676; } \\
\text { 343.0830;193.0504, } \\
401.0522\end{array}$ & 11 \\
\hline 86 & $\begin{array}{l}\text { Conhypoprotocetraric acid or Convirensic } \\
\text { acid }\end{array}$ & $\mathrm{C}_{18} \mathrm{H}_{15} \mathrm{O}_{8}$ & 19.25 & 359.0781 & 359.0767 & 3.9 & $\mathrm{D}$ & $\begin{array}{l}344.0545 ; \\
302.0442\end{array}$ & 3 \\
\hline 87 & 4-O-dimethylbaemycesic acid & $\mathrm{C}_{18} \mathrm{H}_{15} \mathrm{O}_{8}$ & 19.27 & 359.0781 & 359.0767 & 3.9 & d & $\begin{array}{c}\text { 181.0714; } \\
\text { 163.0397; } 137.0236\end{array}$ & $1 ; 2 ; 3 ; 5 ; 6$ \\
\hline 88 & Orsellinic acid Isomer & $\mathrm{C}_{8} \mathrm{H}_{7} \mathrm{O}_{4}$ & 19.45 & 167.0344 & 167.0346 & -1.1974 & A & $\begin{array}{l}123.0440 \\
149.0235 \\
167.0345\end{array}$ & $9 ; 10$ \\
\hline 89 & Lecanoric acid & $\mathrm{C}_{16} \mathrm{H}_{13} \mathrm{O}_{7}$ & 19.51 & 317.0661 & 317.0671 & 0.6 & d & $\begin{array}{l}123.0443 \\
149.0238 \\
357,0626\end{array}$ & $1 ; 2 ; 3 ; 4 ; 5 ; 6$ \\
\hline 90 & Constictic acid isomer & $\mathrm{C}_{19} \mathrm{H}_{13} \mathrm{O}_{10}$ & 19.56 & 401.0509 & 401.0524 & -3.74 & $\mathrm{D}$ & $\begin{array}{l}313.0726 \\
343.0831 \\
255.0622\end{array}$ & 11 \\
\hline 91 & Pentahydroxytetracosanoic acid & $\mathrm{C}_{24} \mathrm{H}_{47} \mathrm{O}_{7}$ & 19.67 & 447.3322 & 447.3336 & -3.1 & $\mathrm{~L}$ & - & $1 ; 3 ; 7 ; 9 ; 10$ \\
\hline 92 & $\begin{array}{l}\text { 2-Methyl-5-hydroxy-6-hydroxymethyl-7- } \\
\text { Methoxychromone }\end{array}$ & $\mathrm{C}_{12} \mathrm{H}_{11} \mathrm{O}_{5}$ & 19.73 & 235.0606 & 235.0615 & -3.8 & C & 181.0504 & 3 \\
\hline $\begin{array}{l}93 \\
94\end{array}$ & $\begin{array}{l}\text { Unknown } \\
\text { Heptahydroxytrioxooctadecanoic acid }\end{array}$ & $\begin{array}{l}\mathrm{C}_{20} \mathrm{H}_{17} \mathrm{O}_{8} \\
\mathrm{C}_{18} \mathrm{H}_{29} \mathrm{O}_{12}\end{array}$ & $\begin{array}{l}19.79 \\
19.86\end{array}$ & $\begin{array}{l}385.0939 \\
437.1664\end{array}$ & $\begin{array}{l}385.0923 \\
437.1645\end{array}$ & $\begin{array}{l}4.1 \\
4.3\end{array}$ & $\overline{\mathrm{L}}$ & 二 & $\begin{array}{c}1 ; 4 ; 5 ; 10 \\
1 ; 4 ; 5 ; 6 ; 7 ; 9 ; 10\end{array}$ \\
\hline 95 & 5,7-Dihydroxy-6-methylphthalide derivative & $\mathrm{C}_{9} \mathrm{H}_{7} \mathrm{O}_{3}$ & 19.88 & 163.0395 & 163.0392 & 1.8 & A & $\begin{array}{l}119.0492 \\
133.0288\end{array}$ & 10 \\
\hline 96 & Criptostictic acid derivative & $\mathrm{C}_{18} \mathrm{H}_{11} \mathrm{O}_{8}$ & 20.04 & 355.0454 & 355.0462 & -2.2 & $\mathrm{D}$ & $\begin{array}{l}\text { 239.0715; } \\
\text { 311.0572; } \\
179.0345\end{array}$ & 7 \\
\hline $\begin{array}{l}97 \\
98\end{array}$ & $\begin{array}{l}\text { Unknow } \\
\text { Unknow }\end{array}$ & $\begin{array}{l}\mathrm{C}_{18} \mathrm{H}_{17} \mathrm{O}_{6} \\
\mathrm{C}_{20} \mathrm{H}_{15} \mathrm{O}_{8}\end{array}$ & $\begin{array}{l}20.08 \\
20.16\end{array}$ & $\begin{array}{l}329.1025 \\
383.0767\end{array}$ & $\begin{array}{l}329.1032 \\
383.0775\end{array}$ & $\begin{array}{l}-2.1 \\
-2.0\end{array}$ & - & - & $9 \begin{array}{c}9 \\
9: 10\end{array}$ \\
\hline 99 & Unknow & $\mathrm{C}_{21} \mathrm{H}_{19} \mathrm{O}_{9}$ & 20.18 & 415.1045 & 415.1029 & 3.9 & - & 二 & $3 ; 5 ; 9 ; 10$ \\
\hline 100 & Unknow & $\mathrm{C}_{28} \mathrm{H}_{23} \mathrm{O}_{11}$ & 20.12 & 535.1240 & 535.1257 & -3.1 & - & - & 3 \\
\hline 101 & Unknow & $\mathrm{C}_{15}^{-} \mathrm{H}_{13}^{-} \mathrm{O}_{3}$ & 20.13 & 241.0872 & 241.0874 & -0.8 & - & - & $1 ; 2$ \\
\hline 102 & Heptahydroxytetraoxoicosanoic acid & $\mathrm{C}_{20} \mathrm{H}_{31} \mathrm{O}_{13}$ & 21.19 & 479.1765 & 479.1746 & 3.9 & $\mathrm{~L}$ & $-\overline{-}$ & 7 \\
\hline 103 & Tetrahydroxytricosanoic acid & $\mathrm{C}_{23} \mathrm{H}_{45} \mathrm{O}_{6}$ & 20.26 & 417.3232 & 417.3216 & 3.9 & $\overline{\mathrm{L}}$ & 403.3073 & $1 ; 3 ; 4 ; 7$ \\
\hline 104 & Tetrahydroxytrioxoundecanoic acid & $\mathrm{C}_{11}^{2} \mathrm{H}_{15} \mathrm{O}_{9}$ & 20.30 & 291.0716 & 291.0699 & 5.8 & $\overline{\mathrm{L}}$ & $\begin{array}{l}-\overline{-} \\
341.0674 ; \\
357.0622\end{array}$ & 8 \\
\hline 105 & Stictic acid & $\mathrm{C}_{19} \mathrm{H}_{13} \mathrm{O}_{9}$ & 20.34 & 385.0560 & 385.0576 & -4.1 & $\mathrm{D}$ & $\begin{array}{c}\text { 297.0774; } \\
313.0721 ; \\
\text { 193.0504; } 269.0826\end{array}$ & 11 \\
\hline $\begin{array}{l}106 \\
107\end{array}$ & $\begin{array}{l}\text { Parietin } \\
\text { Unknow }\end{array}$ & $\begin{array}{l}\mathrm{C}_{16} \mathrm{H}_{11} \mathrm{O}_{5} \\
\mathrm{C}_{24} \mathrm{H}_{47} \mathrm{O}_{11} \mathrm{~N}\end{array}$ & $\begin{array}{r}20.39 \\
\mathrm{~N}_{2} 20.39\end{array}$ & $\begin{array}{l}283.0606 \\
539.3157\end{array}$ & $\begin{array}{l}283.0617 \\
539.3180\end{array}$ & $\begin{array}{l}-3.9 \\
-4.3\end{array}$ & Antraquinone & 179.0345 & $1 ; 2 ; 6$ \\
\hline 108 & Evernic acid isomer & $\mathrm{C}_{17} \mathrm{H}_{15} \mathrm{O}_{7}$ & 20.46 & 331.0818 & 331.0830 & -3.6 & d & $\begin{array}{l}\text { 167.0347; } \\
\text { 123.0447; } 149.0240 \\
\text { 149.0238; }\end{array}$ & 1 \\
\hline 109 & Hypoconstictic acid & $\mathrm{C}_{19} \mathrm{H}_{15} \mathrm{O}_{9}$ & 20.50 & 387.0716 & 387.0732 & -4.1 & $\mathrm{D}$ & $\begin{array}{l}343.0836 ; \\
167.0345 \\
267,0661\end{array}$ & 4 \\
\hline 110 & Cryptostictic acid & $\mathrm{C}_{19} \mathrm{H}_{15} \mathrm{O}_{9}$ & 20.50 & 387.0716 & 387.0725 & -2.3 & $\mathrm{D}$ & $\begin{array}{l}343,0825 \\
311.05067 \\
239.0710\end{array}$ & $7 ; 8$ \\
\hline $\begin{array}{l}111 \\
112\end{array}$ & $\begin{array}{l}\text { Retigeric acid derivative } \\
\text { Retigeric acid B }\end{array}$ & $\begin{array}{l}\mathrm{C}_{30} \mathrm{H}_{43} \mathrm{O}_{7} \\
\mathrm{C}_{30} \mathrm{H}_{45} \mathrm{O}_{6}\end{array}$ & $\begin{array}{l}20.51 \\
20.56\end{array}$ & $\begin{array}{l}515.3009 \\
501.3216\end{array}$ & $\begin{array}{l}515.3025 \\
501.3236\end{array}$ & $\begin{array}{l}-3.1 \\
-4.0\end{array}$ & $\begin{array}{l}\text { Triterpene } \\
\text { Triterpene }\end{array}$ & - & $\begin{array}{l}3 \\
3\end{array}$ \\
\hline 113 & Salazinic acid isomer & $\mathrm{C}_{18} \mathrm{H}_{11} \mathrm{O}_{10}$ & 20.58 & 387.0352 & 387.0368 & -4.13 & $\mathrm{D}$ & $\begin{array}{l}343.0468 \\
299.0565\end{array}$ & 11 \\
\hline $\begin{array}{l}114 \\
115 \\
116\end{array}$ & $\begin{array}{l}\text { Unknown } \\
\text { 9,10-dihydroxyoctadecatrienoic acid } \\
\text { Unknow }\end{array}$ & $\begin{array}{l}\mathrm{C}_{23} \mathrm{H}_{22} \mathrm{O}_{10} \mathrm{~N} \\
\mathrm{C}_{18} \mathrm{H}_{29} \mathrm{O}_{4} \\
\mathrm{C}_{17} \mathrm{H}_{13} \mathrm{O}_{6}\end{array}$ & $\begin{array}{r}\text { N } 20.68 \\
20.69 \\
20.84\end{array}$ & $\begin{array}{l}472.1244 \\
309.2081 \\
313.0712\end{array}$ & $\begin{array}{l}472.1259 \\
309.2066 \\
313.0727\end{array}$ & $\begin{array}{c}-3.2 \\
4.9 \\
-4.8\end{array}$ & $\begin{array}{l}\bar{L} \\
\bar{L}\end{array}$ & 291.1975 & $\begin{array}{c}6 \\
1 ; 2 ; 4 \\
1 ; 2 ; 6\end{array}$ \\
\hline 117 & Pulvinic acid derivative I & $\mathrm{C}_{18} \mathrm{H}_{11} \mathrm{O}_{6}$ & 20.97 & 323.0556 & 323.0556 & 0.0 & $\begin{array}{l}\text { Pulvinic acid y } \\
\text { derivates }\end{array}$ & $\begin{array}{l}\text { 133.0286; } \\
117.0335\end{array}$ & 10 \\
\hline 118 & 9,10,12 Trihydroxytriacontaheptaenoic acid & $\mathrm{C}_{30} \mathrm{H}_{45} \mathrm{O}_{5}$ & 20.99 & 485.3284 & 485.3267 & 3.5 & $\mathrm{~L}$ & - & 3 \\
\hline 119 & 4-0-Demethylbarbatic acid & $\mathrm{C}_{18} \mathrm{H}_{17} \mathrm{O}_{7}$ & 20.99 & 345.0974 & 345.0989 & -4.3 & $\mathrm{~d}$ & $\begin{array}{c}\text { 181.0505; } \\
163.0396 ; 137.0603\end{array}$ & 4 \\
\hline 120 & $\begin{array}{l}\text { Unknow } \\
\text { Methyl orsellinate }\end{array}$ & $\begin{array}{l}\mathrm{C}_{24} \mathrm{H}_{23} \mathrm{O}_{10} \mathrm{~N} \\
\mathrm{C}_{9} \mathrm{H}_{9} \mathrm{O}_{4}\end{array}$ & N 21.02 & $\begin{array}{l}485.1322 \\
181.0502\end{array}$ & $\begin{array}{l}485.1319 \\
181.0501\end{array}$ & 0.6 & $\bar{A}$ & 163.0389 & $1 ; 5$ \\
\hline 122 & Heptahydroxyetraoxoicosanoic acid & $\mathrm{C}_{20} \mathrm{H}_{31} \mathrm{O}_{13}$ & 21.17 & 479.1752 & 479.1765 & -2.7 & $\mathrm{~L}$ & 167.0346 & $1 ; 2 ; 3 ; 4 ; 5 ; 6 ; 9$ \\
\hline 123 & Gyrophoric Acid & $\mathrm{C}_{24} \mathrm{H}_{19} \mathrm{O}_{10}$ & 21.25 & 467.0991 & 467.0978 & 2.8 & d & $\begin{array}{l}\text { 317.0673; } \\
\text { 123.0445; } \\
\text { 149.0238; } \\
403.0681 ; \\
371.0417\end{array}$ & $1 ; 3 ; 4 ; 6 ; 11$ \\
\hline 124 & Galbinic acid & $\mathrm{C}_{20} \mathrm{H}_{13} \mathrm{O}_{11}$ & 21.27 & 429.0458 & 429.0474 & -3.73 & $\mathrm{D}$ & $\begin{array}{l}401.0524 \\
327.0518 \\
149.0239\end{array}$ & 11 \\
\hline 125 & Hyposalazinic acid & $\mathrm{C}_{18} \mathrm{H}_{13} \mathrm{O}_{8}$ & 21.28 & 357.0610 & 357.0623 & -3.6 & $\mathrm{D}$ & $\begin{array}{c}313.0723 ; \\
135.0444 ; 179.0348\end{array}$ & 1 \\
\hline 126 & Hydroxytetracosapentaenoic acid & $\mathrm{C}_{24} \mathrm{H}_{37} \mathrm{O}_{3}$ & 21.42 & 373.2743 & 373.2743 & 0.0 & $\mathrm{~L}$ & $149-$ & 10 \\
\hline 127 & Orsellinic acid isomer & $\mathrm{C}_{8} \mathrm{H}_{7} \mathrm{O}_{4}$ & 21.47 & 167.0347 & 167.0344 & 1.8 & A & $\begin{array}{l}149.0239 \\
123.0443\end{array}$ & $1 ; 4 ; 6$ \\
\hline 128 & Dihydroxyoctadecenoic acid & $\mathrm{C}_{18} \mathrm{H}_{33} \mathrm{O}_{4}$ & 21.48 & 313.2390 & 313.2395 & -1.6 & $\mathrm{~L}$ & & $2 ; 5 ; 6$ \\
\hline 129 & Norstictic acid & $\mathrm{C}_{18} \mathrm{H}_{11} \mathrm{O}_{9}$ & 21.64 & 371.0403 & 371.0417 & -3.77 & $\mathrm{D}$ & $\begin{array}{l}27.0517 ; 227.0716 ; \\
151.0390 ; 243.0297\end{array}$ & 11 \\
\hline $\begin{array}{l}130 \\
131 \\
132\end{array}$ & $\begin{array}{l}\text { Dihydroxyoctadec-6-enoic acid } \\
\text { Loxodinol isomer } \\
\text { EthyI 2,4-dihydroxy-6-n-nonylbenzoate }\end{array}$ & $\begin{array}{l}\mathrm{C}_{18} \mathrm{H}_{33} \mathrm{O}_{4} \\
\mathrm{C}_{25} \mathrm{H}_{29} \mathrm{O}_{9} \\
\mathrm{C}_{18} \mathrm{H}_{27} \mathrm{O}_{4}\end{array}$ & $\begin{array}{l}21.58 \\
21.64 \\
21.65\end{array}$ & $\begin{array}{l}313.2379 \\
473.1812 \\
307.1909\end{array}$ & $\begin{array}{l}313.2379 \\
473.1818 \\
307.1922\end{array}$ & $\begin{array}{l}0.0 \\
-1.2 \\
-4.2\end{array}$ & $\begin{array}{c}\mathrm{L} \\
\mathrm{DE} \\
\mathrm{A}\end{array}$ & $\begin{array}{l}429.1919 \\
263.1659 \\
167.0345\end{array}$ & $\begin{array}{c}10 \\
9 \\
1 ; 2 ; 3 ; 4\end{array}$ \\
\hline 133 & Evernic Acid & $\mathrm{C}_{17} \mathrm{H}_{15} \mathrm{O}_{7}$ & 21.81 & 331.0828 & 331.0818 & 3.0 & d & $\begin{array}{l}167.0345 ; \\
123.0444 ; \\
149,0238 ; \\
355.0460 ; \\
329.0674\end{array}$ & $1 ; 2 ; 3 ; 4 ; 5$ \\
\hline 134 & Protocetraric acid Isomer & $\mathrm{C}_{18} \mathrm{H}_{13} \mathrm{O}_{9}$ & 21.85 & 373.0560 & 373.0573 & -3.48 & $\mathrm{D}$ & $\begin{array}{l}285.0780 \\
311.0567 \\
255 ., 0672\end{array}$ & 11 \\
\hline $\begin{array}{l}135 \\
136\end{array}$ & $\begin{array}{l}\text { Unknown } \\
\text { Strepsilin }\end{array}$ & $\begin{array}{l}\mathrm{C}_{22} \mathrm{H}_{22} \mathrm{O}_{8} \mathrm{~N} \\
\mathrm{C}_{15} \mathrm{H}_{9} \mathrm{O}_{5}\end{array}$ & $\begin{array}{l}21.83 \\
21.89\end{array}$ & $\begin{array}{l}428.1360 \\
269.0450\end{array}$ & $\begin{array}{l}428.1345 \\
269.0462\end{array}$ & $\begin{array}{c}3.5 \\
-4.5\end{array}$ & $\stackrel{-}{\mathrm{DBF}}$ & 225.0554 & $\begin{array}{c}6 \\
2 ; 5\end{array}$ \\
\hline
\end{tabular}


Table 1. Cont.

\begin{tabular}{|c|c|c|c|c|c|c|c|c|c|}
\hline Peak & Tentative Identification & [M-H]- & $\begin{array}{l}\text { Retention } \\
\text { Time (min) }\end{array}$ & $\begin{array}{l}\text { Theoretical } \\
\text { Mass }(\mathrm{m} / \mathrm{z})\end{array}$ & $\begin{array}{l}\text { Measured } \\
\text { Mass }(\mathrm{m} / \mathrm{z})\end{array}$ & $\begin{array}{c}\text { Accuracy } \\
(\mathrm{ppm})\end{array}$ & Metabolite Type & MS Ions $(m / z)$ & Lichen Species * \\
\hline 137 & Unknown & $\mathrm{C}_{30} \mathrm{H}_{29} \mathrm{O}_{4}$ & 21.97 & 453.2066 & 453.2061 & 1.1 & - & - & 3 \\
\hline 138 & Unknown & $\mathrm{C}_{18} \mathrm{H}_{11} \mathrm{O}_{6}$ & 22.01 & 323.0556 & 323.0570 & -4.33 & - & - & 11 \\
\hline 139 & Hexahydroxytrioxooctacosatrienoic acid & $\mathrm{C}_{28} \mathrm{H}_{43} \mathrm{O}_{11}$ & 22.12 & 555.2805 & 555.2841 & -6.4832 & $\mathrm{~L}$ & - & 9 \\
\hline 140 & Nonahydroxyoctacosatetraenoic acid & $\begin{array}{l}\mathrm{C}_{28} \\
\mathrm{H}_{47} \mathrm{O}_{11}\end{array}$ & 22.26 & 559.3124 & 559.3132 & -1.4 & $\mathrm{~L}$ & - & 2 \\
\hline 141 & Unknow & $\begin{array}{l}\mathrm{C}_{48} \\
\mathrm{C}_{28} \mathrm{H}_{41} \mathrm{O}_{9} \mathrm{~N}_{2}\end{array}$ & $\mathrm{~N}_{2} 22.26$ & 549.2849 & 549.2812 & 6.8 & - & - & 3 \\
\hline 142 & Norsolorinic acid & $\mathrm{C}_{20} \mathrm{H}_{17} \mathrm{O}_{7}$ & 22.44 & 369.0974 & 369.0989 & -4.06 & - & - & 11 \\
\hline 143 & Unknow & $\mathrm{C}_{25} \mathrm{H}_{33} \mathrm{O}_{13}$ & 22.46 & 541.1921 & 541.1909 & 2.2 & - & - & $1 ; 2 ; 3 ; 6 ; 10$ \\
\hline 144 & Hydroxytetracosapentaenoic acid derivative & $\mathrm{C}_{24}^{25} \mathrm{H}_{37} \mathrm{O}_{3}$ & 22.61 & 373.2743 & 373.2741 & 0.5 & - & - & 1, 10 \\
\hline 145 & Hydroxytrioxotricosanoic acid & $\mathrm{C}_{23} \mathrm{H}_{39} \mathrm{O}_{6}$ & 22.53 & 411.2747 & 411.2757 & -2.4 & $\mathrm{~L}$ & $\begin{array}{l}-\overline{-} \\
343.0836 \\
163.0396\end{array}$ & 8 \\
\hline 146 & Squamatic acid & $\mathrm{C}_{19} \mathrm{H}_{17} \mathrm{O}_{9}$ & 22.89 & 389.0873 & 389.0886 & -3.3 & d & $\begin{array}{l}\text { 193.0139; } \\
149.0238 ; \\
121.0286\end{array}$ & $1 ; 3 ; 4$ \\
\hline 147 & Picrolichenic acid & $\mathrm{C}_{25} \mathrm{H}_{29} \mathrm{O}_{7}$ & 22.72 & 441.1913 & 441.1926 & -3.0 & Depsones & - & 1 \\
\hline 148 & Heptahydroxydioxohexacosanoic acid & $\mathrm{C}_{26} \mathrm{H}_{47} \mathrm{O}_{11}$ & 22.76 & 535.3118 & 535.3134 & -3.0 & $\mathrm{~L}$ & - & 6 \\
\hline 149 & Unknow & $\mathrm{C}_{30} \mathrm{H}_{45} \mathrm{O}_{4}$ & 22.83 & 469.3319 & 469.3335 & -3.4 & - & - & 3 \\
\hline 150 & 2,2'-Di-O-methylanziaic acid & $\mathrm{C}_{26} \mathrm{H}_{33} \mathrm{O}_{7}$ & 22.85 & 457.2226 & 457.2244 & -3.9 & $\mathrm{~d}$ & 413.2345; & 4 \\
\hline 151 & Dihydroxytetracosahexaenoic acid & $\mathrm{C}_{24} \mathrm{H}_{35} \mathrm{O}_{4}$ & 22.85 & 387.2535 & 387.2552 & -4.4 & $\mathrm{~L}$ & - & 5 \\
\hline 152 & Hydroxyoctadecadienoic acid & $\mathrm{C}_{18}^{24} \mathrm{H}_{31} \mathrm{O}_{3}$ & 22.90 & 295.2273 & 295.2273 & 0.0 & L & - & 10 \\
\hline 153 & Orsellinic acid Isomer & $\mathrm{C}_{8} \mathrm{H}_{7} \mathrm{O}_{4}$ & 22.92 & 167.0344 & 167.0348 & -2.3 & A & $\begin{array}{l}149.0240 \\
123.0445\end{array}$ & 11 \\
\hline 154 & Pulvinic acid & $\mathrm{C}_{18} \mathrm{H}_{11} \mathrm{O}_{5}$ & 22.98 & 307.0606 & 307.0613 & -2.2 & $\begin{array}{c}\text { Pulvinic acid y } \\
\text { derivates }\end{array}$ & $\begin{array}{l}117.0338 ; \\
263.0713 \\
123.0443\end{array}$ & 9 \\
\hline 155 & 4-0-Demethylbarbatic acid & $\mathrm{C}_{18} \mathrm{H}_{17} \mathrm{O}_{7}$ & 23.02 & 345.0974 & 345.0986 & -3.5 & d & $\begin{array}{l}123.0443 \\
137.0237 \\
181.0502 \\
313.0726 \\
181.0502\end{array}$ & 1 \\
\hline 156 & Psoromic acid & $\mathrm{C}_{18} \mathrm{H}_{13} \mathrm{O}_{8}$ & 23.06 & 357.0610 & 357.0626 & -4.4 & $\mathrm{D}$ & $\begin{array}{l}179.0347 ; \\
327.0520 ; \\
269.0826 ; \\
285.0776 \\
149.0238\end{array}$ & 11 \\
\hline 157 & Methylgyrophoric acid & $\mathrm{C}_{25} \mathrm{H}_{21} \mathrm{O}_{10}$ & 23.15 & 481.1135 & 481.1147 & -2.5 & d & $\begin{array}{l}\text { 123.0442; } \\
\text { 167.0346; } \\
317.0671 \\
\text { 149.0239; }\end{array}$ & $1 ; 4$ \\
\hline 158 & Evernic acid isomer & $\mathrm{C}_{17} \mathrm{H}_{15} \mathrm{O}_{7}$ & 23.22 & 331.0818 & 331.0832 & -4.2 & d & $\begin{array}{l}123.0443 \\
167.0346 \\
105.0337\end{array}$ & 11 \\
\hline 159 & Skyrin & $\mathrm{C}_{30} \mathrm{H}_{17} \mathrm{O}_{10}$ & 23.28 & 537.0822 & 537.0840 & -3.4 & Anthraquinones & - & 3 \\
\hline 160 & Angardianic acid & $\mathrm{C}_{19} \mathrm{H}_{35} \mathrm{O}_{4}$ & 23.36 & 327.2543 & 327.2547 & -1.2 & Acids & $\begin{array}{l}283.2649 ; \\
309.2081\end{array}$ & $2 ; 4$ \\
\hline 161 & Pentadecatetraenoic acid & $\mathrm{C}_{15} \mathrm{H}_{21} \mathrm{O}_{2}$ & 23.38 & 233.1542 & 233.1545 & -1.2 & L & - & $9 ; 10$ \\
\hline 162 & 9-hydroxyoctadecatrienoic acid & $\mathrm{C}_{18}$ & 23.45 & 293.2117 & 293.2130 & -4.4 & $\mathrm{~L}$ & 277.2180 & 6 \\
\hline 163 & Unknow & $\mathrm{C}_{18} \mathrm{H}_{15} \mathrm{O}_{7}$ & 23.53 & 343.0818 & 343.0824 & -1.7 & - & - & 9 \\
\hline 164 & Pulvinic acid derivative II & $\mathrm{C}_{19} \mathrm{H}_{13} \mathrm{O}_{5}$ & 23.68 & 321.0763 & 321.0770 & -2.1 & $\begin{array}{l}\text { Pulvinic acid y } \\
\text { derivates }\end{array}$ & 117.0337 & $9 ; 10 ; 11$ \\
\hline 165 & Pulvinic acid & $\mathrm{C}_{18} \mathrm{H}_{11} \mathrm{O}_{5}$ & 23.77 & 307.0606 & 307.0620 & -4.5 & $\begin{array}{l}\text { derrvates } \\
\text { Pulvinic acid y } \\
\text { derivates }\end{array}$ & $\begin{array}{l}\text { 263.0720; } \\
\text { 117.0339 } \\
371.0784\end{array}$ & 11 \\
\hline 166 & Furfuric acid isomer & $\mathrm{C}_{28} \mathrm{H}_{23} \mathrm{O}_{12}$ & 23.82 & 551.1190 & 551.1197 & -1.2 & $\mathrm{D}$ & $\begin{array}{l}\text { 371.0784; } \\
\text { 193.0504; } \\
\text { 179.0347; } \\
\text { 207.0297; } \\
\text { 193.0504; }\end{array}$ & 8 \\
\hline 167 & Unknow & $\mathrm{C}_{26} \mathrm{H}_{47} \mathrm{O}_{5} \mathrm{~N}_{2}$ & $\mathrm{~N}_{2} 23.82$ & 467.3485 & 467.3492 & $-3,9$ & - & - & 3 \\
\hline $\begin{array}{l}168 \\
169\end{array}$ & $\begin{array}{l}\text { Unknow } \\
\text { Unknow }\end{array}$ & $\begin{array}{l}\mathrm{C}_{30}^{-} \mathrm{H}_{27} \mathrm{O}_{6} \\
\mathrm{C}_{30} \mathrm{H}_{25} \mathrm{O}_{6}\end{array}$ & $\begin{array}{l}23.89 \\
24.01\end{array}$ & $\begin{array}{l}483.1808 \\
481.1651\end{array}$ & $\begin{array}{l}483.1820 \\
481.1663\end{array}$ & $\begin{array}{l}-2,5 \\
-2,5\end{array}$ & $\begin{array}{l}- \\
-\end{array}$ & 二 & $\begin{array}{l}1 \\
1\end{array}$ \\
\hline 170 & Unknow & $\mathrm{C}_{15} \mathrm{H}_{13} \mathrm{O}_{3}$ & 24.02 & 241.0872 & 241.0872 & 0,0 & - & - & 1 \\
\hline 171 & Trihydroxyheptacosa pentaenoic acid & $\mathrm{C}_{27} \mathrm{H}_{43} \mathrm{O}_{5}$ & 24.05 & 447.3110 & 447.3127 & -3.8 & $\mathrm{~L}$ & $\overline{-} \overline{0}$ & 8 \\
\hline 172 & Barbatic Acid & $\mathrm{C}_{19} \mathrm{H}_{19} \mathrm{O}_{7}$ & 24.26 & 359.1141 & 359.1131 & 2.8 & $\mathrm{~d}$ & $\begin{array}{l}\text { 137.0603; } \\
\text { 163.0396; } \\
\text { 181.0509 }\end{array}$ & $1 ; 4$ \\
\hline 173 & Hydroxytrioxodocosanoic acid & $\mathrm{C}_{22} \mathrm{H}_{37} \mathrm{O}_{6}$ & 24.29 & 397.2590 & 397.2601 & -2.8 & L & $101 .-$ & 8 \\
\hline 174 & Thamnolic acid isomer & $\mathrm{C}_{19} \mathrm{H}_{15} \mathrm{O}_{11}$ & 24.41 & 419.0614 & 419.0630 & -3.8 & $\mathrm{~d}$ & $\begin{array}{l}375.0730 \\
\text { 167.0344; } \\
209.0455 \\
181.0503\end{array}$ & 11 \\
\hline 175 & Orsenillic acid derivated II & $\mathrm{C}_{8} \mathrm{H}_{7} \mathrm{O}_{4}$ & 24.73 & 167.0344 & 167.0347 & -1.80 & - & $\begin{array}{l}149.0239 \\
1230444\end{array}$ & 11 \\
\hline 176 & Unknow & $\mathrm{C}_{26} \mathrm{H}_{33} \mathrm{O}_{8}$ & 24.73 & 473.2190 & 473.2175 & 3.2 & - & $1200+4+$ & 2 \\
\hline 177 & Lobaric acid & $\mathrm{C}_{25} \mathrm{H}_{27} \mathrm{O}_{8}$ & 24.81 & 455.1706 & 455.1718 & -2.6 & $\mathrm{D}$ & $\begin{array}{l}\text { 411.1824; } \\
367.1811\end{array}$ & 1 \\
\hline $\begin{array}{l}178 \\
179\end{array}$ & $\begin{array}{l}\text { Unknow } \\
\text { Unknow }\end{array}$ & $\begin{array}{l}\mathrm{C}_{22} \mathrm{H}_{27} \mathrm{O}_{7} \\
\mathrm{C}_{30} \mathrm{H}_{41} \mathrm{O}_{8}\end{array}$ & $\begin{array}{l}24.97 \\
25.27\end{array}$ & $\begin{array}{l}403.1770 \\
529.2819\end{array}$ & $\begin{array}{l}403.1757 \\
529.2801\end{array}$ & $\begin{array}{l}3.2 \\
3.4\end{array}$ & - & - & $\begin{array}{l}2 \\
4\end{array}$ \\
\hline 180 & Hypothamnolic acid & $\mathrm{C}_{19} \mathrm{H}_{17} \mathrm{O}_{10}$ & 25.49 & 405.0822 & 405.0832 & -2.5 & $\mathrm{~d}$ & $\begin{array}{l}209.0456 ; \\
181.0499\end{array}$ & 1 \\
\hline 181 & Unknow & $\mathrm{C}_{25} \mathrm{H}_{11} \mathrm{O}_{7}$ & 25.53 & 423.0505 & 423.0497 & 1.9 & - & 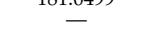 & 4 \\
\hline 182 & Pulvinic acid derivative III & $\mathrm{C}_{19} \mathrm{H}_{13} \mathrm{O}_{5}$ & 25.67 & 321.0763 & 321.0777 & -4.3 & $\begin{array}{c}\text { Pulvinic acid y } \\
\text { derivates }\end{array}$ & 117.0338 & 11 \\
\hline 183 & Dihydroxyicosahexaenoic acid & $\mathrm{C}_{20} \mathrm{H}_{27} \mathrm{O}_{4}$ & 26.02 & 331.1909 & 331.1925 & -4.8 & L & - & 11 \\
\hline 184 & Usnic acid & $\mathrm{C}_{18} \mathrm{H}_{15} \mathrm{O}_{7}$ & 26.05 & 343.0818 & 343.0831 & -3.8 & DBF & $\begin{array}{l}231.0658 ; \\
328.0585 ;\end{array}$ & $3 ; 4 ; 6 ; 7 ; 8$ \\
\hline $\begin{array}{l}185 \\
186\end{array}$ & $\begin{array}{l}\text { Nephromopsic acid orRoccellaric acid } \\
\text { Unknow }\end{array}$ & $\begin{array}{l}\mathrm{C}_{19} \mathrm{H}_{33} \mathrm{O}_{4} \\
\mathrm{C}_{28} \mathrm{H}_{25} \mathrm{O}_{5} \mathrm{~N}\end{array}$ & N $\begin{array}{r}26.32 \\
26.87\end{array}$ & $\begin{array}{l}325.2392 \\
455.1733\end{array}$ & $\begin{array}{l}325.2379 \\
455.1723\end{array}$ & $\begin{array}{c}4.0 \\
2.20\end{array}$ & Acids & $\begin{array}{l}259.0604 \\
281.2494\end{array}$ & $\begin{array}{l}3 ; 4 \\
11\end{array}$ \\
\hline 187 & Perlatolic acid & $\mathrm{C}_{25} \mathrm{H}_{31} \mathrm{O}_{7}$ & 26.98 & 443.2070 & 443.2078 & -1.8 & $\mathrm{~d}$ & 205.0867; & $7 ; 8$ \\
\hline 188 & Caperatic acid & $\mathrm{C}_{21} \mathrm{H}_{37} \mathrm{O}_{7}$ & 28.14 & 401.2539 & 401.2549 & -2.4 & Acids & $\begin{array}{l}223.0973 \\
255.2327\end{array}$ & 8 \\
\hline 189 & Atranorin & $\mathrm{C}_{19} \mathrm{H}_{17} \mathrm{O}_{8}$ & 29.64 & 373.0923 & 373.0937 & -3.75 & $\mathrm{~d}$ & $\begin{array}{l}\text { 177.0192; } \\
\text { 163.0397 }\end{array}$ & $9 ; 11$ \\
\hline
\end{tabular}

${ }^{*}$ Identified by addition experiments with a genuine compound. A = Aromatic compound; $\mathrm{L}=\mathrm{Lipid} ; \mathrm{D}=$ depsidone; $\mathrm{d}=$ depside; $\mathrm{DE}=$ diphenilether; $\mathrm{DBF}=$ dibenzofurane. $\mathrm{C}=$ Chromone. $\quad * * \mathbf{1}=S$. cf. andina; $\mathbf{2}=S$. cf. hypoglabra; $\mathbf{3}=$ S. cordillerana; $\mathbf{4}=$ S. cf. gyalocarpa; $\mathbf{5}=$ S. leucoblepharis; $\mathbf{6}=$ S. parahumboldtii; $\mathbf{7}=$ S. impressula $; \mathbf{8}=$ S. ocaniesnsis; $\mathbf{9}=$ S. speudosylvatica $; \mathbf{1 0}=$ S. luteocyphelata $; \mathbf{1 1}=$ S. lineariloba. 


\subsubsection{Saturated Organic Acids}

Peak 1 was tentatively identified as gluconic acid $\left(\mathrm{C}_{6} \mathrm{H}_{11} \mathrm{O}_{7}\right)$, peak 3 as manitol, peak 4 as arabic acid, peak 5 as citric acid and peak 10 as its isomer isocitric acid $\left(\mathrm{C}_{6} \mathrm{H}_{7} \mathrm{O}_{7}\right)$, peak 7 as 4-ethyl-2-ethylisophthalic acid $\left(\mathrm{C}_{10} \mathrm{H}_{9} \mathrm{O}_{4}\right)$ with peak 2 as its isomer 2-ethylisophthalic acid, peak 15 as the derivative 2-hydroxyisophthalic acid, peak 17 was identified as 4-Odemethylglomellic acid $\left(\mathrm{C}_{24} \mathrm{H}_{25} \mathrm{O}_{9}\right)$, peak 30 as 1,5-pentanedicarboxylic acid $\left(\mathrm{C}_{7} \mathrm{H}_{11} \mathrm{O}_{4}\right)$ and peak 44 as 2,4-dicarboxy-3-hydroxy-5-methoxytoluene $\left(\mathrm{C}_{10} \mathrm{H}_{9} \mathrm{O}_{6}\right)$

\subsubsection{Small Phenolic Compounds}

Peaks 14 and 16 were identified as trihydroxy benzalaldehyde $\left(\mathrm{C}_{7} \mathrm{H}_{5} \mathrm{O}_{4}\right)$ and 2,4-dihydroxy benzaldehyde $\left(\mathrm{C}_{7} \mathrm{H}_{5} \mathrm{O}_{3}\right)$, peak 25 as 5,7-dihydroxy-6-methylphthalide $\left(\mathrm{C}_{9} \mathrm{H}_{7} \mathrm{O}_{4}\right)$, peak 39 as metil-2,6-dihidroxibenzoate $\left(\mathrm{C}_{8} \mathrm{H}_{7} \mathrm{O}_{4}\right)$, peak 51 as 2,4-dihydroxy benzaldehyde $\left(\mathrm{C}_{7} \mathrm{H}_{5} \mathrm{O}_{3}\right)$, peak 53 as 4-ethoxy-3-formyl-2-hydroxy-6-methylbenzoic acid $\left(\mathrm{C}_{11} \mathrm{H}_{11} \mathrm{O}_{5}\right)$, peak 95 ,7dihydroxy-6-methylphthalide derivative $\left(\mathrm{C}_{9} \mathrm{H}_{7} \mathrm{O}_{3}\right)$, peak 132 as ethyl 2,4-dihydroxy-6-nnonylbenzoate $\left(\mathrm{C}_{18} \mathrm{H}_{27} \mathrm{O}_{4}\right)$.

\subsubsection{Typical Lichenic Phenolic Compounds (Depsides, Depsidones and Anthraquinones)}

Several compounds were identified as the typical types of compounds occurring in lichens in our Sticta species. Peak 20, with a deprotonated molecule at $m / z: 413.1569$, was identified as grayanic acid $\left(\mathrm{C}_{23} \mathrm{H}_{25} \mathrm{O}_{7}\right)$, peak 23 was identified as atranol $\left(\mathrm{C}_{8} \mathrm{H}_{7} \mathrm{O}_{3}\right)$ [25], peak 32 as didechlorolecideoidin $\left(\mathrm{C}_{17} \mathrm{H}_{13} \mathrm{O}_{7}\right)$ [36] showing diagnostic fragments at $m / z$ : 209.0456; 285.0776; 151.0396; 179.0347 and 123.0443, peak 34 was identified as orsellinic acid $\left(\mathrm{C}_{8} \mathrm{H}_{7} \mathrm{O}_{4}\right)$, peak 37 as nor $8^{\prime}$-methylconstictic acid $\left(\mathrm{C}_{21} \mathrm{H}_{19} \mathrm{O}_{11}\right)$, showing a diagnostic daughter fragment at $m / z$ : 209.0455, peak 40 as hypostictic acid isomer $\left(\mathrm{C}_{19} \mathrm{H}_{15} \mathrm{O}_{8}\right)$, peak 42 as fumarprotocetraric acid derivative $\left(\mathrm{C}_{17} \mathrm{H}_{11} \mathrm{O}_{6}\right)$, peak 52 as consalizinic acid derivative I $\left(\mathrm{C}_{19} \mathrm{H}_{13} \mathrm{O}_{11}\right.$, with diagnostic daughter ions at $\mathrm{m} / \mathrm{z}$ : 373.0573; 387.0373; 225.0406 and 177.0193, and peak 55 as consalizinic acid derivative II, with ions at $m / z$ : 401.0524; 417.0474 and 373.0574), peak 56 and 57 as an cynodontin, citreorosein isomer or consalizinic acid derivative I isomer, respectively, peak 59 as 1,4,5,6,8-pentahydroxy-3-ethylanthraquinone $\left(\mathrm{C}_{15} \mathrm{H}_{9} \mathrm{O}_{7}\right)$, peak 62 , with an ion at $\mathrm{m} / \mathrm{z}$ : 403.0681 was identified as a haemathamnolic acid isomer $\left(\mathrm{C}_{19} \mathrm{H}_{15} \mathrm{O}_{10}\right)$ and peak 64 as constictic acid $\left(\mathrm{C}_{19} \mathrm{H}_{13} \mathrm{O}_{10}\right)$, peak 63 as a fumarprotocetraric acid derivative $\left(\mathrm{C}_{17} \mathrm{H}_{11} \mathrm{O}_{6}\right)$, peak 65 as hypostictic acid isomer $\left(\mathrm{C}_{19} \mathrm{H}_{15} \mathrm{O}_{8}\right)$, producing daughter ions at $m / z$ : 327.0883, 195.0664 and 179.0347 peak 66 as terphenylquinones thelephoric acid $\left(\mathrm{C}_{18} \mathrm{H}_{7} \mathrm{O}_{8}\right)$ and peak 67 as methylstictic acid $\left(\mathrm{C}_{20} \mathrm{H}_{15} \mathrm{O}_{9}\right.$, ions at 371.0779 and 193.0504). Peak 68 was tentatively identified as $8^{\prime}$-metilconstictic acid isomer $\left(\mathrm{C}_{21} \mathrm{H}_{19} \mathrm{O}_{11}\right)$, and peak 69 as protocetraric acid $\left(\mathrm{C}_{18} \mathrm{H}_{13} \mathrm{O}_{9}\right)$ [29], peak 70 as hypoconstictic acid $\left(\mathrm{C}_{19} \mathrm{H}_{15} \mathrm{O}_{9}\right)$, peak 77 as menegazziaic acid $\left(\mathrm{C}_{18} \mathrm{H}_{13} \mathrm{O}_{9}\right)$ [37] with ionic fragments at $\mathrm{m} / \mathrm{z}$ : 311.0570; $255.0666 ; 329.0679$, peak 78 as norstictic acid $\left(\mathrm{C}_{18} \mathrm{H}_{11} \mathrm{O}_{9}\right.$ ions at $m / z: 327.0526 ; 151.0396$ and 123.0444), peak 80 as the antioxidant agent physodalic acid [35] $\left(\mathrm{C}_{20} \mathrm{H}_{15} \mathrm{O}_{10}, \mathrm{MS}^{2}\right.$ peaks at $\mathrm{m} / \mathrm{z}$ : 359.0417; 315.0520; 343.0832; 387.0367; 373.0573 and 401.0525$)$, peak 82 as derivative methyl 8-hydroxy-4-O-demethylbarbatate $\left(\mathrm{C}_{19} \mathrm{H}_{19} \mathrm{O}_{9}\right)$, peak 83 as $12,13,15$ trihydroxy-9-octadecenoic acid $\left(\mathrm{C}_{18} \mathrm{H}_{33} \mathrm{O}_{5}\right)$, peak 84 as the cytotoxic compound haemoventosin $\left(\mathrm{C}_{15} \mathrm{H}_{11} \mathrm{O}_{7}\right)$ [38], with ions at $259.0619,231.0667,189.0560$, peak 86 as conhypoprotocetraric acid or convirensic acid $\left(\mathrm{C}_{18} \mathrm{H}_{15} \mathrm{O}_{8}\right)$ [29], peak 87 as 4-O-dimethylbaeomycesic acid $\left(\mathrm{C}_{18} \mathrm{H}_{15} \mathrm{O}_{8}, 181.0714 ; 163.0397\right.$ and 137.0236), a methyl derivative of baeomycesic acid [39], peak 88 as orsellinic acid isomer $\left(\mathrm{C}_{8} \mathrm{H}_{7} \mathrm{O}_{4}, 123.0440 ; 149.0235\right)$, peak 89 as lecanoric acid $\left(\mathrm{C}_{16} \mathrm{H}_{13} \mathrm{O}_{7}, 167.0345 ; 123.0443 ; 149.0238\right)$ [23], peak 90 as constictic acid isomer $\left(\mathrm{C}_{19} \mathrm{H}_{13} \mathrm{O}_{10}\right)$, peak 92 with a deprotonated molecule at $m / z: 235.0615$ and daughter ion at $m / z: 181.0504$ was identified as 2-methyl-5-hydroxy-6-hydroxymethyl-7-methoxychromone $\left(\mathrm{C}_{12} \mathrm{H}_{11} \mathrm{O}_{5}\right)$, peak 96 was identified as criptostictic acid derivative $\left(\mathrm{C}_{18} \mathrm{H}_{11} \mathrm{O}_{8}\right)$, peak 105 as stictic acid $\left(\mathrm{C}_{19} \mathrm{H}_{13} \mathrm{O}_{9}\right)$ [25], peak 106 as the typical lichen anthraquinone parietin $\left(\mathrm{C}_{16} \mathrm{H}_{11} \mathrm{O}_{5}\right)$, while peak 108 was identified as evernic acid isomer $\left(\mathrm{C}_{17} \mathrm{H}_{15} \mathrm{O}_{7}\right)$, peak 109 as hypoconstictic acid and peak 110 as cryptostictic acid [40] $\left(\mathrm{C}_{19} \mathrm{H}_{15} \mathrm{O}_{9}\right.$, diagnostic daughter ions at $\mathrm{m} / \mathrm{z}$ : 267.0661; $343.0825,311.05067$ and 239.0710$)$. peak 113 as salazinic acid isomer $\left(\mathrm{C}_{18} \mathrm{H}_{11} \mathrm{O}_{1}\right)$, peak 117 
with a deprotonated molecule at $m / z: 323.0556$ was identified as pulvinic acid derivative I $\left(\mathrm{C}_{18} \mathrm{H}_{11} \mathrm{O}_{6}\right)$, producing ions at $m / z$ : 133.0286; 117.0335. Peak 119 , with a parent ion at $m / z$ : 345.0989, was identified as 4-O-demethylbarbatic acid $\left(\mathrm{C}_{18} \mathrm{H}_{17} \mathrm{O}_{7}\right)$ [29], peak 121 as methyl orsellinate [37] and peak 123 as gyrophoric acid. Peak 125, producing fragments at $m / z$ : 313.0723; 135.0444 and 179.0348 , was identified as hyposalazinic acid $\left(\mathrm{C}_{18} \mathrm{H}_{13} \mathrm{O}_{8}\right)$ [37], peak 127 as an isomer of orsellinic acid [23], peak 124 as galbinic acid $\left(\mathrm{C}_{20} \mathrm{H}_{13} \mathrm{O}_{11}, 403.0681\right.$; 371.0417; 401.0524; 327.0518 and 149.0239) [37], peak 129 as norstictic acid $\left(\mathrm{C}_{18} \mathrm{H}_{11} \mathrm{O}_{9}\right.$, $27.0517 ; 227.0716 ; 151.0390 ; 243.0297)$ [1,2], peak 133 as evernic acid $\left(\mathrm{C}_{17} \mathrm{H}_{15} \mathrm{O}_{7}\right)$ [25], peak 131 as loxodinol isomer $\left(\mathrm{C}_{25} \mathrm{H}_{29} \mathrm{O}_{9}\right)$, peak 136 as the dibenzophenone strepsilin $\left(\mathrm{C}_{15} \mathrm{H}_{9} \mathrm{O}_{5}\right)$, peak 146 as squamatic acid and peak 147 as the depsone picrolichenic acid $\left(\mathrm{C}_{25} \mathrm{H}_{29} \mathrm{O}_{7}\right)$, peak 144 and 145 as hydroxytetracosapentaenoic acid derivative $\left(\mathrm{C}_{24} \mathrm{H}_{37} \mathrm{O}_{3}\right)$ and hydroxytrioxotricosanoic acid $\left(\mathrm{C}_{23} \mathrm{H}_{39} \mathrm{O}_{6}\right)$, respectively. Peak 150 , with an ion at $\mathrm{m} / \mathrm{z}: 457.2244$, was identified as 2,2' -di-O-methylanziaic acid $\left(\mathrm{C}_{26} \mathrm{H}_{33} \mathrm{O}_{7}\right)$ [41], peak 154 was determined to be pulvinic acid, $\left(\mathrm{C}_{18} \mathrm{H}_{11} \mathrm{O}_{5}\right.$, ions at $m / z$ : 117.0338; 263.0713), peak 164 as a pulvinic acid derivative $\left(\mathrm{C}_{19} \mathrm{H}_{13} \mathrm{O}_{5}\right)$, peak 164 as a pulvinic acid derivative of $321.0770\left(\mathrm{C}_{19} \mathrm{H}_{13} \mathrm{O}_{5}\right)$, and peak 165 as another isomer of pulvinic acid $\left(\mathrm{C}_{18} \mathrm{H}_{11} \mathrm{O}_{5}\right)$, while peak 155 was identified as 4-O-demethylbarbatic acid $\left(\mathrm{C}_{18} \mathrm{H}_{17} \mathrm{O}_{7}\right.$, diagnostic ions at $m / z$ : $\left.123.0443 ; 137.0237 ; 181.0502\right)$. Peak 156 was determined as soromic acid (ions at $m / z: 313.0726 ; 181.0502 ; 179.0347 ; 327.0520$; 269.0826 and 285.0776), peak 157 as methylgyrophoric acid $\left(\mathrm{C}_{25} \mathrm{H}_{21} \mathrm{O}_{10}\right.$, diagnostic ions at $m / z$ : 149.0238; 123.0442; 167.0346 and 317.0671), and peak 158 as evernic acid isomer. Similarly, peak 159 was identified as anthraquinones skyrin $\left(\mathrm{C}_{30} \mathrm{H}_{17} \mathrm{O}_{10}\right)$, peak 160 as angardianic acid $\left(\mathrm{C}_{19} \mathrm{H}_{35} \mathrm{O}_{4}\right)$ [42], peak 166, with an ion at $m / z: 551.1197$, was identified as furfuric acid isomer $\left(\mathrm{C}_{28} \mathrm{H}_{23} \mathrm{O}_{12}\right.$, producing fragments at $m / z$ : 371.0784; 193.0504; 179.0347; 207.0297 and 193.0504), peak 172 as barbatic acid $\left(\mathrm{C}_{19} \mathrm{H}_{19} \mathrm{O}_{7}\right)$ [25], peak 174 as thamnolic acid, peak 175 as orsenillic acid derivative II $\left(\mathrm{C}_{8} \mathrm{H}_{7} \mathrm{O}_{4}\right)$, peak 177 as lobaric acid $\left(\mathrm{C}_{25} \mathrm{H}_{27} \mathrm{O}_{8}\right.$ with ions at $m / z$ : 411.1824; 367.1811), peak 180 as hypothamnolic acid $\left(\mathrm{C}_{19} \mathrm{H}_{17} \mathrm{O}_{10}\right.$ with ions at $m / z$ : 209.0456; 181.0499) [43], peak 184 as usnic acid $\left(\mathrm{C}_{18} \mathrm{H}_{15} \mathrm{O}_{7}\right)$ [25], peak 185 as either nephromopsic acid or roccellaric acid $\left(\mathrm{C}_{19} \mathrm{H}_{33} \mathrm{O}_{4}\right)$ [44], and finally, peak 187 was identified as the cytotoxic compound perlatolic acid [45], peak 188 as the antibacterial compound caperatic acid [46] and peak 189 as atranorin.

\subsubsection{Terpenes}

Peak 112 was identified as retigeric acid $\mathrm{B}\left(\mathrm{C}_{30} \mathrm{H}_{45} \mathrm{O}_{6}\right)$, while peak 111 with an ion at $m / z$ : 515.3025 was tentatively identified as a retigeric acid derivative $\left(\mathrm{C}_{30} \mathrm{H}_{43} \mathrm{O}_{7}\right)$.

\subsubsection{Lipids}

Oxylipins polyunsaturated fatty acids are an important dietary compounds, and can be found in edible fruits by HPLC orbitrap mass spectrometry [47] and also can be found in useful plants [48] and lichens [49]. In this study, several fatty acids including saturated fats and oxylipins were found using this technique in Sticta lichens. Peak 33, with a parent ion at $m / z$ : 555.3047, was identified as decahydroxyoxopentacosanoic acid $\left(\mathrm{C}_{25} \mathrm{H}_{47} \mathrm{O}_{13}\right)$; peak 43, with a parent ion at $m / z$ : 187.0977, was determined to be 4,5-dihydroxy-2-nonenoic acid $\left(\mathrm{C}_{9} \mathrm{H}_{15} \mathrm{O}_{4}\right)$; peak 72 as 12,13,15-trihydroxy-9-octadecenoic acid $\left(\mathrm{C}_{18} \mathrm{H}_{15} \mathrm{O}_{5}\right)$, while peaks 91 and 94 were determined as pentahydroxytetracosanoic acid $\left(\mathrm{C}_{24} \mathrm{H}_{47} \mathrm{O}_{7}\right)$ and heptahydroxytrioxooctadecanoic acid $\left(\mathrm{C}_{18} \mathrm{H}_{29} \mathrm{O}_{12}\right)$, respectively. In the same manner, peaks 102-104 were identified as heptahydroxytetraoxoicosanoic acid $\left(\mathrm{C}_{20} \mathrm{H}_{31} \mathrm{O}_{13}\right)$, tetrahydroxytricosanoic acid $\left(\mathrm{C}_{23} \mathrm{H}_{45} \mathrm{O}_{6}\right)$, and tetrahydroxytrioxoundecanoic acid $\left(\mathrm{C}_{11} \mathrm{H}_{15} \mathrm{O}_{9}\right)$, respectively. Peak 115 was assigned as 9,10-dihydroxyoctadecatrienoic acid $\left(\mathrm{C}_{18} \mathrm{H}_{29} \mathrm{O}_{4}\right)$ and peak 118 as 9,10,12 trihydroxytriacontaheptaenoic acid; peak 122 as eptahydroxyetraoxoicosanoic acid $\left(\mathrm{C}_{20} \mathrm{H}_{31} \mathrm{O}_{13}\right)$; peak 126 as hydroxytetracosapentaenoic acid $\left(\mathrm{C}_{24} \mathrm{H}_{37} \mathrm{O}_{3}\right)$; and peak 128 as dihydroxyoctadecenoic acid $\left(\mathrm{C}_{18} \mathrm{H}_{33} \mathrm{O}_{4}\right)$. Peak 130 was tentatively identified as dihydroxyoctadec-6-enoic acid $\left(\mathrm{C}_{18} \mathrm{H}_{33} \mathrm{O}_{4}\right)$; peak 134 as a protocetraric acid isomer $\left(\mathrm{C}_{18} \mathrm{H}_{13} \mathrm{O}_{9}\right)$ [29]; peak 139 as hexahydroxytrioxooctacosatrienoic acid $\left(\mathrm{C}_{28} \mathrm{H}_{43} \mathrm{O}_{11}\right)$; peak 140 as nonahydroxyoctacosatetraenoic acid $\left(\mathrm{C}_{28} \mathrm{H}_{47} \mathrm{O}_{11}\right)$; peak 142 as norsolorinic acid 
$\left(\mathrm{C}_{20} \mathrm{H}_{17} \mathrm{O}_{7}\right)$; peak 148 as heptahydroxydioxohexacosanoic acid $\left(\mathrm{C}_{26} \mathrm{H}_{47} \mathrm{O}_{11}\right)$; peak 151 as dihydroxytetracosahexaenoic acid $\left(\mathrm{C}_{24} \mathrm{H}_{35} \mathrm{O}_{4}\right)$; and peak 152 as hydroxyoctadecadienoic acid $\left(\mathrm{C}_{18} \mathrm{H}_{31} \mathrm{O}_{3}\right)$. In the same manner, peak 161 and 162 were attributed to pentadecatetraenoic acid and 9-hydroxyoctadecatrienoic acid, respectively. Finally, peak 171 was identified as trihydroxyheptacosa pentaenoic acid; peak 173 as hydroxytrioxodocosanoic acid; and peak 183 as dihydroxyicosahexaenoic acid $\left(\mathrm{C}_{20} \mathrm{H}_{27} \mathrm{O}_{4}\right)$.

In this study, we worked on 11 species of the genus Sticta from Colombia and Chile. It should be noted that the species were collected in different ecosystems and environmental conditions in South America. The analyses includes 189 compounds, 41 of which had not yet been identified, of which the most representative are gluconic acid (1), citric acid (5), 2-Ethylisophthalic acid (13), orsellinic acid (34), lecanoric acid (89), stictic acid (105), parietin (106), gyrophoric acid (123) and usnic acid (184) (Figure 3). It should be noted that none of the identified and unidentified compounds are present simultaneously in all 11 species.<smiles>O=C(O)[C@H](O)[C@@H](O)[C@H](O)[C@H](O)CO</smiles>

Peak 1, Gluconic Acid<smiles>O=C(O)CC(O)(CC(=O)O)C(=O)O</smiles><smiles>CCc1c(C(=O)O)cccc1C(=O)O</smiles><smiles>Cc1cc(O)cc(O)c1C(=O)O</smiles><smiles>Cc1cc(OC(=O)c2c(C)cc(O)cc2O)cc(C)c1C(=O)O</smiles><smiles>COc1ccc2c(c1C=O)Oc1c(c(C)c(O)c3c1C(O)OC3=O)OC2=O</smiles>

\section{Peak 34, Orsellinic acid Peak 89, Lecanoric acid Peak 105, Stictic acid}<smiles>COc1cc(O)c2c(c1)C(=O)c1cc(C)cc(O)c1C2=O</smiles><smiles>CC(=O)C1=C(O)C=C2Oc3c(C(C)=O)c(O)c(C)c(O)c3C2(C)C1=O</smiles>

Peak 106, Parietin

\section{Peak 123, Gyrophoric acid}

Peak 184, Usnic acid

Figure 3. Structures of some representative compounds found in Sticta lichens.

\subsubsection{Distance and Phylogenetic Analysis}

We found that $69 / 189$ (37\%) chemical characters and 7/16 (44\%) morphological characters were parsimony informative. Optimally retained trees had a minimum parsimony score of 256 (chemical compounds) and 87 (morphology). The chemical compounds tree showed higher consistency and retention indexes and lower homoplasy $(\mathrm{CI}=0.966, \mathrm{RI}=0.786)$ than the morphology tree $(\mathrm{CI}=0.738 ., \mathrm{RI}=0.531)$. 
In general, maximum-parsimony strict consensus trees from morphological and chemical characters were not consistent with each other and did not recover the same relationship between taxa (Figure 4), whereas the morphological tree recovers most of the evolutionary relationships (positions in phylogeny) documented in the published molecular phylogeny of Colombian Sticta [50] and the chemical-compound tree mirrors the geographic clusters of collected samples exactly.
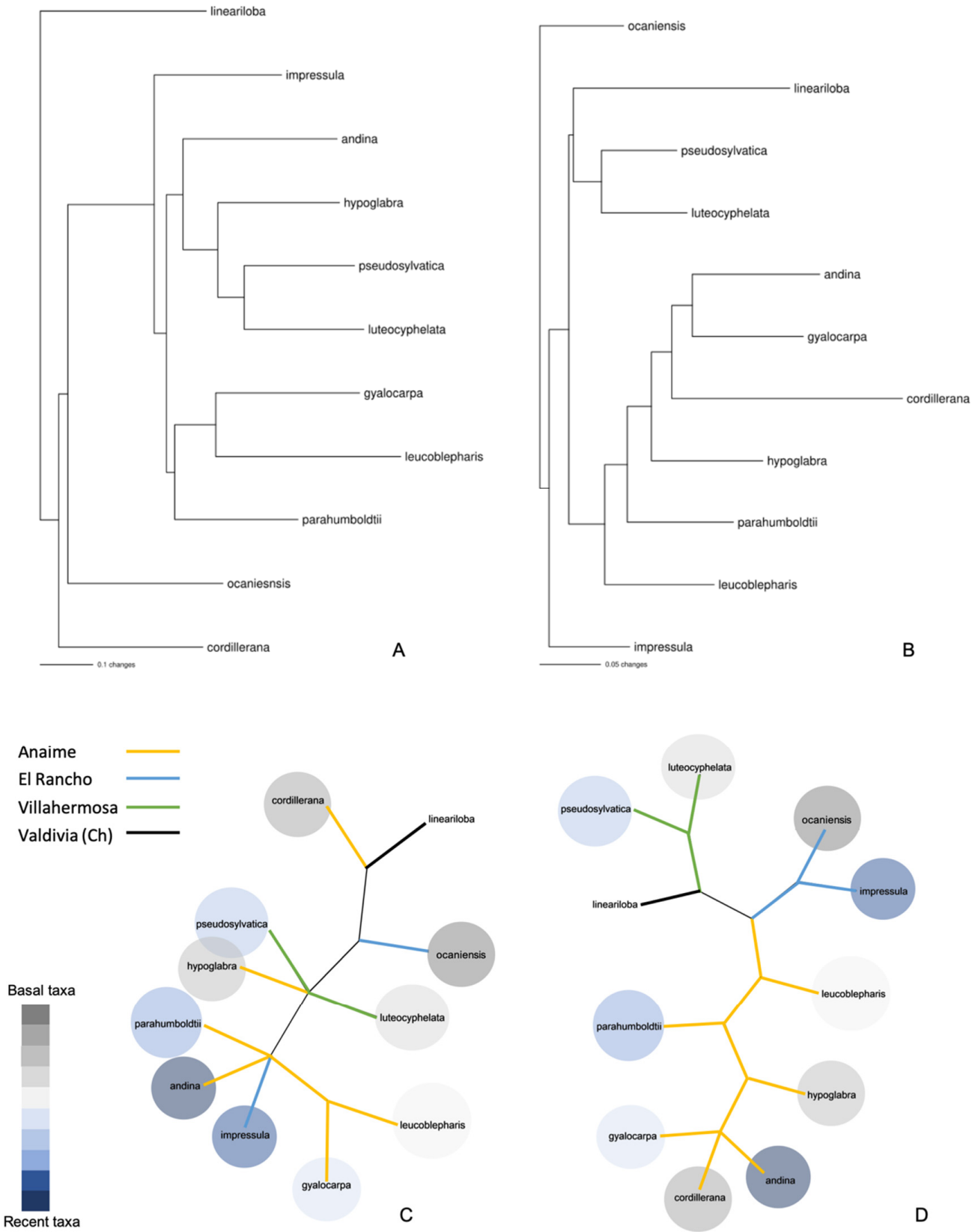

Figure 4. Neighbor joining trees $(\mathbf{A}, \mathbf{B})$ and unrooted strict consensus trees from maximum parsimony analysis $(\mathbf{C}, \mathbf{D})$ with morphological traits $(\mathbf{A}, \mathbf{C})$ and chemical compounds $(\mathbf{B}, \mathbf{D})$ in some Colombian species of Sticta. Colored lines represent localities from collected specimens. Colored circles represent their position in the molecular phylogeny of Moncada et al. (2014); grayish colors in the color scale refer to more basal taxa, whereas bluish colors are more recent taxa. Although S. lineariloba occurs in Colombia, the specimen in this study was collected in Valdivia (Chile). Independent of the grouping method, trees built based on chemical compounds recovered the geographic relationships, while trees built with morphological traits resemble the molecular phylogeny of Colombian Sticta. 
In the last two decades, the study of secondary metabolites in lichens has represented an input for the determination of specimens in different complex groups, through their intervention in taxonomic keys. These compounds are mostly aromatic derivatives such as depsides, depsidones, dibenzofurans, dibenzoquinones and usnic acid among others that derive from the biochemical pathways generated by malonic, mevalonic and shikimic acids [51]. The morphological data used in the present analysis of phylogenetic relationships within species of Sticta demonstrate the relevance of morphological traits in lichen taxonomy. Nevertheless, the chemical characters offer the possibility of an alternative comparison, independent from the morphology-based classification system [52]. In this study, the analysis of chemical traits recovered more geographic than ancestor-descendant relationships among taxa. These results enrich the discussion of the role of the local environment on lichen adaptation through the actions of natural selection on biochemical pathways.

In other studies, groupings based on chemical compounds such as the case of $S$. cf ocaniensis, S. cf. pseudolobaria, and S. canariensis, were consistent with the known molecular phylogeny of Sticta, as was the case with S. pulmonarioides and S. cf. weigelia [53]. For the genus Cetrelia, an assessment of the composition of secondary metabolites allowed for a confirmation of the presence of species only reported in America in Europe, as in the case of $C$. chicitae. Metabolite composition has also facilitated the confirmation of new species for the genus by chemical fingerprinting, which contrasts the phenotypic plasticity of some morphological characters used for identification [54]. In the genus Psoroma, an analysis of the distribution of secondary metabolites has validated the presence of chemical markers unique to the group, and the presumed description of subgenera by taxa heterogeneity, causing spatial segregation [55].

On the other hand, in the genus Cladonia, there is evidence of the use of chemotaxonomic methods to determine and differentiate phylogenetically related species (C. arbuscula, C. borealis, C. chlorophaea, C. coccifera, C. coniocraea, C. cornuta, C. fimbriata, C. mitis, C. monomorpha, C. pyxidate, C. rangiferina, C. stellaris, and C. stygia), which contain chemical markers exclusive to the group [56]. In this way, the morphological-anatomical data are complemented, and discriminatory characters are provided to distinguish the species. In the genus Blastenia, reduced chemotypes are also reported in some lineages with particular genetic characteristics and distribution [57].

Currently, the process of chemotaxonomic discrimination analysis in lichen groups requires reinforcement with complementary techniques, such as the use of pigments derived from anthraquinone-type compounds in specimens of the family Teloschistaceae (Pyrenodesmia sensu lato) [58] and optical-sensor profiles for metabolic profiling in species of the genera Cladonia, Stereocaulon, Lichina, Collema and Peltigera [59]. In addition, advances in analytical chemistry and mass spectrometry have allowed for a greater specificity in the elaboration of bioactive compounds profiles, which, together with modern DNAsequencing techniques and the extension of morphological descriptions as a "polyphasic approach", provide objectivity in the delimitation of lichen species [60,61]. However, due to the wide variation in lichen chemotypes, the use of new compounds such as fatty acids is proposed in chemotaxonomy and phylogeny analyses, and in building an understanding of molecular-complex communication and compound biosynthetic pathways [62].

\section{Materials and Methods}

\subsection{Chemicals}

Ultrapure water was obtained from a water purification system brand Millipore (MilliQ Merck Millipore, Santiago, Chile). Analytical reagents were all purchased from Sigma Aldrich Co. (Santiago, Chile). Ethanol, Methanol, formic acid, acetone, and acetonitrile were of chromatographic grade for HPLC analysis. Analytical lichen standards (purity: 98\% by HPLC) were purchased from Sigma-Aldrich Chemical Company (Santiago, Chile). 


\subsection{Lichen Material}

The lichen specimens S. pseudosylvatica Moncada \& Suárez (35 g) and S. luteocyphellata Moncada \& Lücking ( $28 \mathrm{~g}$ ) were collected by Olimpo García Beltrán and Alfredo Torres Benítez in 2018 in Villahermosa, in the department of Tolima-Colombia, at the farm La Estrella $\left(5^{\circ} 02^{\prime} 48.63^{\prime \prime} \mathrm{N}-75^{\circ} 07^{\prime} 37.98^{\prime \prime} \mathrm{W}\right)$. The species $S$. cf. andina Moncada \& Lücking (31 g), S. cf. hypoglabra Moncada \& Lücking (42 g), S. cordillerana Gyeln (37 g), S. cf. gyalocarpa (Nyl.) (29 g), S. leucoblepharis (Nyl.) Tuck. \& Mont (34 g). y S. parahumboldtii Moncada \& Lücking ( $40 \mathrm{~g}$ ) were collected by Alfredo Torres Benítez and Emmanuel Campos in 2018 in the Semillas de Agua Civil Society Nature Reserve in the Anaime páramo $\left(4^{\circ} 15^{\prime} 18.09^{\prime \prime} \mathrm{N}-73^{\circ} 33^{\prime} 23.27^{\prime \prime} \mathrm{W}\right)$ and the species S. impressula (Nyl.) Zahlbr (29 g) and S. ocaniensis (33 g) Moncada \& Simijaca were collected by Alfredo Torres Benítez and María Rivera Montalvo in 2017 in the "Combéima river basin", Ibagué-Tolima, Colombia $\left(4^{\circ} 36^{\prime} 02.35^{\prime \prime} \mathrm{N}-75^{\circ} 19^{\prime} 50.45^{\prime \prime} \mathrm{W}\right)$. All voucher specimens were deposited in the herbarium of Universidad Distrital Francisco José de Caldas (Colombia) and Prof. Alejandra Suárez Corredor confirmed their identity.

\subsection{Preparation of the Sample for Analyses}

Fresh samples were weighed and frozen for two days at $-80^{\circ} \mathrm{C}$. Then, the samples were taken to a freeze-evaporation system (Model 7670541 FreeZone 2.5 Liter Labconco Freeze Dry Systems) and all the water contained in the original product was removed by freeze-evaporation cycles. A total of $3 \mathrm{~g}$ of each dried lichen was macerated with methanol (3 times, $30 \mathrm{~mL}$ each time, 3 days/extraction). The solutions were concentrated to obtain $11 \mathrm{mg}$ of extract from S. pseudosylvatica; $9 \mathrm{mg}$ S. luteocyphellata; $14 \mathrm{mg} \mathrm{S.} \mathrm{cf.} \mathrm{andina;} 12 \mathrm{mg}$ S. cf. hypoglabra; $10 \mathrm{mg}$ S. cordillerana; $9 \mathrm{mg}$ S. cf. gyalocarpa; $13 \mathrm{mg}$ S. leucoblepharis; $13 \mathrm{mg}$ S. parahumboldtii; $8 \mathrm{mg} \mathrm{S}$. impressula and $9 \mathrm{mg} \mathrm{S}$. ocaniensis, respectively. Then, the lichen extracts were processed individually for HPLC-MS analyses (redissolved in methanol at a concentration of $1 \mathrm{mg} / \mathrm{mL}$ for the analyses).

\subsection{Instrument}

A Thermo Scientific Ultimate 3000 UHPLC with a PDA (photodiode array detector) detector controlled by Chromeleon 7.2 Software (Thermo Fisher Scientific, Waltham, MA, USA) in conjunction with a Thermo high resolution Q-Exactive focus mass spectrometer (Thermo, Bremen, Germany) were used for analysis. The chromatographic system was coupled to the MS using a type II heated electrospray ionization source. Nitrogen obtained (purity $>99.999 \%$ ) from a nitrogen generator (Genius NM32LA, Peak Scientific, Billerica, MA, USA) was employed as both the collision and damping gas. Mass calibration for Orbitrap was performed once a day, in both negative and positive modes, to ensure working mass 5 ppm of accuracy. Sodium dodecyl sulfate, caffeine, N-butylamine, buspirone hydrochloride, and taurocholic acid sodium salt (Sigma Aldrich, Saint Louis, MO, USA) plus Ultramark 1621 (Alpha Aezar, Stevensville, MI, USA), a phosphazine fluorinated solution, was the standard mixture used to calibrate the mass spectrometer. These compounds were dissolved in a mixture of acetic acid, acetonitrile, water, and methanol (Merck, Darmstadt, Germany) and were infused using a Chemyx Fusion 100 syringe pump, XCalibur 2.3 software and Trace Finder 3.2 (Thermo Fisher Scientific, San José, CA, USA), which were used for control and data processing. Q Exactive 2.0 SP 2 from Thermo Fisher Scientific was used to control the mass spectrometer. The lichens extracts were individually redissolved in methanol (at a concentration of $1 \mathrm{mg} / \mathrm{mL}$ ), each solution was filtered (PTFE filter, Merck) and then 10 microliters were injected in the UHPLC instrument for UHPLC-MS analysis. XCalibur 2.3 software (Thermo Fisher Scientific, Bremen, Germany) and Trace Finder 3.2 (Thermo Fisher Scientific, San José, CA, USA) were used for UHPLC control and data processing, respectively. Q Exactive 2.0 SP 2 from Thermo Fisher Scientific was used to control the mass spectrometer. 


\subsection{LC Parameters}

Liquid chromatography on a UHPLC C-18 column (Acclaim, $150 \mathrm{~mm} \times 4.6 \mathrm{~mm}$ ID, $2.5 \mu \mathrm{m}$, Thermo Fisher Scientific, Bremen, Germany) was performed as reported previously. The mobile phases were $1 \%$ formic aqueous solution, (A) methanol $1 \%$ formic acid (B) and acetonitrile $1 \%$ formic acid $(C)$. The gradient program time were as follows: $0.00 \mathrm{~min}, 18 \mathrm{~B}$, $75 \mathrm{C} ; 5.00 \mathrm{~min}, 18 \mathrm{~B}, 75 \mathrm{C} ; 15.00 \mathrm{~min}, 40 \mathrm{~B}, 60 \mathrm{C} ; 20.00 \mathrm{~min}$. B, $100 \mathrm{C}$; and $12 \mathrm{~min}$ for column equilibration at starting conditions.

\subsection{MS Parameters}

The HESI parameters were as follows: sheath gas-flow rate of 75 units; aux. gas unit flow rate of 20; capillary temperature of $400^{\circ} \mathrm{C}$; aux gas heater temperature of $500{ }^{\circ} \mathrm{C}$; spray voltage of $2500 \mathrm{~V}$ (for ESI-); and S lens RF level of 30. Full scan data in both the positive and negative modes were acquired at a resolving power of 70,000 FWHM (full width half maximum) at $m / z 200$. For the compounds of interest, a scan range of $m / z 100-1000$ was chosen; the automatic gain control (AGC) was set at $3 \times 10^{6}$ and the injection time set to $200 \mathrm{~ms}$. Scan-rate was set at 2 scans s$^{-1}$. External calibration was performed using a calibration solution in the positive and negative modes. For confirmation purposes, a targeted MS/MS analysis was performed using the mass inclusion list, with a $30 \mathrm{~s}$ time window, with the Orbitrap spectrometer operating both in the positive and negative mode at $17,500 \mathrm{FWHM}(\mathrm{m} / \mathrm{z} 200)$. The AGC target was set to $2 \times 10^{5}$, with the max. injection time of $20 \mathrm{~ms}$. The precursor ions were filtered by the quadrupole, which operates at an isolation window of $\mathrm{m} / \mathrm{z} 2$. The fore vacuum, high vacuum and ultrahigh vacuum were maintained at approximately 2 mbar, from $10^{5}$ and below $10^{10}$ mbar, respectively. Collision energy (HCD cell) was operated at $30 \mathrm{kv}$. Detection was based on calculated exact mass and on retention time of target compounds, as shown in Table 1. The mass tolerance window was set to $5 \mathrm{ppm}$ for the two modes for most compounds.

\subsection{Similarity and Phylogenetic Analyses}

We carried out a phylogenetic study to analyze if the chemical compounds found in Sticta specimens recovered a phylogenetic signal consistent with the current taxonomic relationships in the genera. First, we built two character-state matrices. One matrix included the 189 chemical compounds reported in this paper, and the other encompassed 16 morphological traits (Suppl. Tables S1 and S2). Then, the compounds were coded as binary characters (presence/absence), whereas morphological traits were coded as multistate characters. All character states receive the same weight and were set as unordered. Next, exploratory Neighbor-Joining distance trees were built. After that, we used a maximum parsimony phylogenetic approach to perform an exhaustive search of optimal trees. A maximum of 100 trees were retained after evaluating ca. 34 million trees per matrix. Trees were unrooted given the absence of descriptions of chemical compounds and morphological traits for potential outgroups. Then, the consistency and retention indexes were calculated. Finally, we obtained the strict consensus of optimal trees. All the procedures were performed in PAUP 4a168 for mac.

\section{Conclusions}

Eleven lichens of the Sticta genera from two different country zones were phytochemically investigated. More scientific data on chemistry is presented for these interesting lichens that can significantly increase the knowledge and potential for sustainable applications and industrial interest. This valuable natural-product biomass has potential applications in food, medicine, biotechnology, pharmaceuticals, and cosmetics, with many possible applications from food-conserving agents to anticancer biomaterials. The morphological data used in the present analysis of phylogenetic relationships within genus of Sticta demonstrate the relevance of morphological traits in lichen taxonomy. Nevertheless, chemical characters for chemotaxonomic studies offer the possibility of an alternative comparison, independent from the morphology-based classification system. In this study, 
chemical traits' analysis recovered more geographic than ancestor-descendant relationships among taxa, and these results enriched the discussion of the role of the local environment on lichen adaptation through natural selection acting on biochemical pathways.

Supplementary Materials: The following supporting information can be downloaded at: https: / / www.mdpi.com/article/10.3390/metabo12020156/s1, Table S1: Description of character and character states used in the present study of Sticta, Table S2: Character state matrix of morphological traits used to recover phylogenetic relationships in species of Sticta.

Author Contributions: M.J.S., O.G.-B., C.A. conceived and designed the experiments; A.T.-B. performed lichens characterization, L.A., B.S., S.A.M.S. and E.S. performed the LC MS experiments and organized the data of HPLC/MS, M.M.-P. performed the calculations of phylogenetic studies and their chemotaxonomic relationships. All authors wrote the paper, and read and approved the final manuscript. All authors have read and agreed to the published version of the manuscript.

Funding: O.G.-B. Thank funding from the Ministry of Science, Technology and Innovation, the Ministry of Education, the Ministry of Industry, Commerce and Tourism, and ICETEX, Programme Ecosistema Científico-Colombia Científica, from the Francisco José de Caldas Fund, Grand RCFP44842-212-2018; M.J.S. received financial support from Fondecyt, Chile (Grant 1220075) and INACh RT 16-17, while C.A. received funding from Fondecyt Regular 1190314.

Institutional Review Board Statement: Not applicable.

Informed Consent Statement: Not applicable.

Data Availability Statement: Data is contained within the article or Supplementary Material, but raw Thermo HPLC profiles of the plant or other data can be available on author's request.

Conflicts of Interest: The authors declare no conflict of interest. The funding sponsors had no role in the design of the study; in the collection, analyses, interpretation of data; in the writing of the manuscript, and in the decision to publish the results.

\section{References}

1. Nash, T. Lichen Biology, 2nd ed.; Cambridge University Press: Cambridge, MA, USA, 2008.

2. Armaleo, D.; Clerc', P. Lichen chimeras: DNA analysis suggests that one fungus forms twp morphotypes. Exp. Mycol. 1991, 15, 1-10. [CrossRef]

3. Sanders, W.B. Composite lichen thalli of sticta sp. from Brazil, with morphologically similar lobes containing either a chlorobiont or a cyanobiont layer. Symbiosis 2001, 31, 47-55.

4. Henskens, F.L.; Green, T.G.A.; Wilkins, A. Cyanolichens can have both cyanobacteria and green algae in a common layer as major contributors to photosynthesis. Ann. Bot. 2012, 110, 555-563. [CrossRef] [PubMed]

5. Galloway, D.J. Studies on the lichen genus Sticta (Schreber) Ach.: I. Southern South American species. Lichenol 1994, $26,223-282$. [CrossRef]

6. Galloway, D.J. Studies on the lichen genus Sticta (Schreber) Ach.: V*. Australian species. Bryophyt. Divers. Evol. 1998, 15, 117-160. [CrossRef]

7. Galloway, D.J. Studies on the lichen genus Sticta (Schreber) Ach. IV. New Zealand species. Lichenologist 1997, 29, 105-168. [CrossRef]

8. Moncada, B.; Aguirre, J.; Lücking, R. Ecogeografía del género Sticta (ascomycota liquenizados: Lobariaceae) en Colombia. Rev. Biol. Trop. 2014, 62, 266-281. [CrossRef]

9. Moncada, B.; Luecking, R.K.; Lumbsch, H.T. Rewriting the evolutionary history of the lichen genus Sticta (Ascomycota: Peltigeraceae subfam. Lobarioideae) in the Hawaiian Islands. Plant Fungal Syst. 2020, 65, 95-119. [CrossRef]

10. Moncada, B.; Lücking, R. Ten new species of Sticta and counting: Colombia as a hot spot for unrecognized diversification in a conspicuous macrolichen genus. Phytotaxa 2012, 74, 1-29. [CrossRef]

11. Moncada, B.; Mercado-Díaz, J.A.; Magain, N.; Hodkinson, B.P.; Smith, C.W.; Bungartz, F.; Pérez-Pérez, R.E.; Gumboski, E.; Sérusiaux, E.; Lumbsch, H.T.; et al. Phylogenetic diversity of two geographically overlapping lichens: Isolation by distance, environment, or fragmentation? J. Biogeogr. 2021, 48, 676-689. [CrossRef]

12. Mercado-Díaz, J.A.; Lücking, R.; Moncada, B.; Widhelm, T.J.; Lumbsch, H.T. Elucidating species richness in lichen fungi: The genus Sticta (Ascomycota: Peltigeraceae) in Puerto Rico. Taxon 2020, 69, 851-891. [CrossRef]

13. Sepahvand, A.; Studzińska-Sroka, E.; Ramak, P.; Karimian, V. Usnea sp.: Antimicrobial potential, bioactive compounds, ethnopharmacological uses and other pharmacological properties; a review article. J. Ethnopharmacol. 2021, 268, 113656. [CrossRef] [PubMed] 
14. Calcott, M.J.; Ackerley, D.F.; Knight, A.; Keyzers, R.A.; Owen, J.G. Secondary metabolism in the lichen symbiosis. Chem. Soc. Rev. 2018, 47, 1730-1760. [CrossRef] [PubMed]

15. Corbett, B.R.E.; Young, H.J. Lichens and fungi. Part III. Structural elucidation of $15 \alpha, 22$-dihydroxyhopane from Sticta billardierii Del. Chem. Soc. C Org. 1966, 1564-1567. [CrossRef]

16. Chin, W.J.; Corbett, R.E.; Heng, C.K.; Wilkins, A.L. Lichens and fungi. Part XI. Isolation and structural elucidation of a new group of triterpenes from Sticta coronata, S. colensoi, and S. flavicans. J. Chem. Soc. Perkin Trans. 1 1973, 14, 1437-1446. [CrossRef]

17. Piovano, M.; Chamy, M.C.; Garbarino, J.A.; Quilhot, W. Secondary metabolites in the genus Sticta (lichens). Biochem. Syst. Ecol. 2000, 28, 589-590. [CrossRef]

18. Zhang, H.J.; Guo, H.F.; Lou, H.X. Secondary metabolites from the Chinese lichen Sticta nylanderiana A. Z. Biochem. Syst. Ecol. 2006, 34, 760-762. [CrossRef]

19. Liang, Z.; Sorribas, A.; Sulzmaier, F.J.; Jiménez, J.I.; Wang, X.; Sauvage, T.; Yoshida, W.Y.; Wang, G.; Ramos, J.W.; Williams, P.G Stictamides A-C, MMP12 inhibitors containing 4-amino-3-hydroxy-5- phenylpentanoic acid subunits. J. Org. Chem. 2011, 76, 3635-3643. [CrossRef]

20. Le Pogam, P.; Schinkovitz, A.; Legouin, B.; Le Lamer, A.C.; Boustie, J.; Richomme, P. Matrix-free UV-laser desorption ionization mass spectrometry as a versatile approach for accelerating dereplication studies on lichens. Anal. Chem. 2015, 87, 10421-10428. [CrossRef]

21. Musharraf, S.G.; Kanwal, N.; Thadhani, V.M.; Choudhary, M.I. Rapid identification of lichen compounds based on the structurefragmentation relationship using ESI-MS/MS analysis. Anal. Methods 2015, 7, 6066-6076. [CrossRef]

22. Simirgiotis, M.J.; Quispe, C.; Areche, C.; Sepúlveda, B. Phenolic compounds in chilean mistletoe (quintral, Tristerix tetrandus) analyzed by UHPLC-Q/Orbitrap/MS/MS and its antioxidant properties. Molecules 2016, 21, 245. [CrossRef] [PubMed]

23. Cornejo, A.; Salgado, F.; Caballero, J.; Vargas, R.; Simirgiotis, M.; Areche, C. Secondary metabolites in Ramalina terebrata detected by UHPLC/ESI/MS/MS and identification of parietin as tau protein inhibitor. Int. J. Mol. Sci. 2016, 17, 1303. [CrossRef]

24. Castro, O.N.; Benites, J.; Rodilla, J.; Santiago, J.C.; Simirgiotis, M.; Sepulveda, B.; Areche, C. Metabolomic analysis of the lichen Everniopsis trulla using ultra high performance liquid chromatography-quadrupole-orbitrap mass spectrometry (UHPLC-Q-OTMS). Chromatographia 2017, 80, 967-973. [CrossRef]

25. Torres-Benítez, A.; Rivera-Montalvo, M.; Sepúlveda, B.; Castro, O.N.; Nagles, E.; Simirgiotis, M.J.; Garciá-Beltrán, O.; Areche, C. Metabolomic analysis of two Parmotrema lichens: P. robustum (Degel.) Hale and P. andinum (Mull. rg.) hale using UHPLC-ESI-OTMS-MS. Molecules 2017, 22, 1861. [CrossRef]

26. Salgado, F.; Albornoz, L.; Cortéz, C.; Stashenko, E.; Urrea-Vallejo, K.; Nagles, E.; Galicia-Virviescas, C.; Cornejo, A.; Ardiles, A.; Simirgiotis, M.; et al. Secondary metabolite profiling of species of the genus Usnea by UHPLC-ESI-OT-MS-MS. Molecules 2018, 23, 54. [CrossRef]

27. Jiménez-González, A.; Quispe, C.; Bórquez, J.; Sepúlveda, B.; Riveros, F.; Areche, C.; Nagles, E.; García-Beltrán, O.; Simirgiotis, M.J. UHPLC-ESI-ORBITRAP-MS analysis of the native Mapuche medicinal plant palo negro (Leptocarpha rivularis DC.-Asteraceae) and evaluation of its antioxidant and cholinesterase inhibitory properties. J. Enzyme Inhib. Med. Chem. 2018, 23, 54. [CrossRef] [PubMed]

28. Areche, C.; Fernandez-Burgos, R.; Terrones, T.C.D.; Simirgiotis, M.; García-Beltrán, O.; Borquez, J.; Sepulveda, B. Mulinum crassifolium Phil; two new mulinanes, gastroprotective activity and metabolomic analysis by UHPLC-orbitrap mass spectrometry. Molecules 2019, 24, 1673. [CrossRef]

29. Parrot, D.; Jan, S.; Baert, N.; Guyot, S.; Tomasi, S. Comparative metabolite profiling and chemical study of Ramalina siliquosa complex using LC-ESI-MS/MS approach. Phytochemistry 2013, 89, 114-124. [CrossRef]

30. Ly, H.D.; Vo, T.N.; Duong, T.H.; Nguyen, T.D.; Nguyen, K.P.P. A new depside and two new diphenyl ether compounds from the lichen Ramalina farinacea (L.). Ach. Phytochem. Lett. 2015, 11, 146-150. [CrossRef]

31. Alam, M.A.; Khatoon, R.; Huda, S.; Ahmad, N.; Sharma, P.K. Biotechnological Applications of Lichens. In Lichen-Derived Products John Wiley and Sons: Hoboken, NJ, USA, 2020; pp. 203-219.

32. Nguyen, T.T.; Nallapaty, S.; Rao, G.S.N.K.; Koneru, S.T.; Annam, S.S.P.; Tatipamula, V.B. Evaluating the in vitro activity of depsidones from Usnea subfloridana Stirton as key enzymes involved in inflammation and gout. Pharm. Sci. 2021, 27, 291-296. [CrossRef]

33. Zhao, Y.; Wang, M.; Xu, B. A comprehensive review on secondary metabolites and health-promoting effects of edible lichen. J. Funct. Foods 2020, 80, 104283. [CrossRef]

34. Nguyen, T.T.H.; Dinh, M.H.; Chi, H.T.; Wang, S.L.; Nguyen, Q.V.; Tran, T.D.; Nguyen, A.D. Antioxidant and cytotoxic activity of lichens collected from Bidoup Nui Ba National Park, Vietnam. Res. Chem. Intermed. 2019, 45, 33-49. [CrossRef]

35. Aoussar, N.; Laasri, F.E.; Bourhia, M.; Manoljovic, N.; Mhand, R.A.; Rhallabi, N.; Ullah, R.; Shahat, A.A.; Noman, O.M.; Nasr, F.A.; et al. Phytochemical analysis, cytotoxic, antioxidant, and antibacterial activities of lichens. Evid. Based Complement. Altern. Med. 2020, 2020, 1-11. [CrossRef] [PubMed]

36. Elix, J.; Elix, J.; Venables, D.; Venables, D.; Lumbsch, H.; Lumbsch, H.; Brako, L.; Brako, L. Further new metabolites from lichens Aust. J. Chem. 1994, 47, 1619. [CrossRef]

37. Sultana, N.; Afolayan, A.J. A new depsidone and antibacterial activities of compounds from Usnea undulata Stirton. J. Asian Nat. Prod. Res. 2011, 13, 1158-1164. [CrossRef] 
38. Le Pogam, P.; Le Lamer, A.C.; Siva, B.; Legouin, B.; Bondon, A.; Graton, J.; Jacquemin, D.; Rouaud, I.; Ferron, S.; Obermayer, W.; et al. Minor pyranonaphthoquinones from the apothecia of the lichen Ophioparma ventosa. J. Nat. Prod. 2016, 79, 1005-1011. [CrossRef]

39. Goel, M.; Dureja, P.; Rani, A.; Uniyal, P.L.; Laatsch, H. Isolation, characterization and antifungal activity of major constituents of the himalayan Lichen Parmelia reticulata tayl. J. Agric. Food Chem. 2011, 59, 2299-2307. [CrossRef]

40. Asplund, J.; Gauslaa, Y. Content of secondary compounds depends on thallus size in the foliose lichen Lobaria pulmonaria. Lichenologist 2007, 39, 273-278. [CrossRef]

41. Elix, J.A.; Jariangprasert, S.; Archer, A.W. New Pertusaria (lichenized Ascomycota) from Australia and Thailand. Telopea 2008, 12, 263-272. [CrossRef]

42. Brodo, I.M.; Tønsberg, T. Opegrapha halophila (Opegraphaceae), a new lichen species from coastal British Columbia, Canada, and Alaska, USA. Bryologist 2019, 122, 457-462. [CrossRef]

43. Xiang, W.J.; Wang, Q.Q.; Ma, L.; Hu, L.H. $\beta$-Orcinol-type depsides from the lichen Thamnolia vermicularis. Nat. Prod. Res. 2013, 27, 804-808. [CrossRef] [PubMed]

44. Nallasivam, J.L.; Fernandes, R.A. A protecting-group-free synthesis of (+)-nephrosteranic, (+)-protolichesterinic, (+)nephrosterinic, (+)-phaseolinic, (+)-rocellaric acids and (+)-methylenolactocin. Org. Biomol. Chem. 2017, 15, 708-716. [CrossRef] [PubMed]

45. Bogo, D.; Honda, N.K.; Alcantara, G.B.; Brandão, L.F.G.; Aléssio, G.F.; Guimarães, R.D.C.A.; Matos, M.D.F.C. Cytotoxic activity of compounds from lichens of the cerrado biome in Brazil. Orbital 2020, 12, 7-16. [CrossRef]

46. Dieu, A.; Mambu, L.; Champavier, Y.; Chaleix, V.; Sol, V.; Gloaguen, V.; Millot, M. Antibacterial activity of the lichens Usnea florida and Flavoparmelia caperata (Parmeliaceae). Nat. Prod. Res. 2020, 34, 3358-3362. [CrossRef] [PubMed]

47. Simirgiotis, M.J.; Ramirez, J.E.; Schmeda, H.G.; Kennelly, E.J. Bioactive coumarins and HPLC-PDA-ESI-ToF-MS metabolic profiling of edible queule fruits (Gomortega keule), an endangered endemic Chilean species. Food Res. Int. 2013, 54, 532-543. [CrossRef]

48. Brahmi-Chendouh, N.; Piccolella, S.; Nigro, E.; Hamri-Zeghichi, S.; Madani, K.; Daniele, A.; Pacifico, S. Urtica dioica L. leaf chemical composition: A never-ending disclosure by means of HR-MS/MS techniques. J. Pharm. Biomed. Anal. 2021, 195, 113892. [CrossRef]

49. Gouveia-Figueira, S.; Danielsson, K.; Fowler, C.J. Changes in proportions of linoleic acid-derived oxylipins in oral lichen planus. Acta Derm. Venereol. 2019, 99, 1051-1052. [CrossRef]

50. Moncada, B.; Lücking, R.; Suárez, A. Molecular phylogeny of the genus Sticta (lichenized Ascomycota: Lobariaceae) in Colombia Fungal Divers. 2014, 64, 205-231. [CrossRef]

51. Swofford, D.L. PAUP*. Phylogenetic Analysis Using Parsimony ( ${ }^{*}$ and Other Methods); Version 4; Sinauer Associates: Sunderland, MA, USA, 2003.

52. Culberson, W.L. Chemosystematics and Ecology of Lichen-Forming Fungi. Annu. Rev. Ecol. Syst 1970, 1, 153-170. [CrossRef]

53. Mittermeier, V.K.; Schmitt, N.; Volk, L.P.M.; Suárez, J.P.; Beck, A.; Eisenreich, W. Metabolic profiling of alpine and ecuadorian lichens. Molecules 2015, 20, 18047-18065. [CrossRef]

54. Farkas, E.; Biró, B.; Varga, N.; Sinigla, M.; Lőkös, L. Analysis of lichen secondary chemistry doubled the number of Cetrelia W.L. Culb. \& C.F. Culb. species (Parmeliaceae, lichenised Ascomycota) in Hungary. Cryptogamie. Mycologie 2021, 42, 1-16. [CrossRef]

55. Quilhot, W.; Piovano, M.; Arancibia, H. Studies on Chilean Lichens, XII. Chemotaxonomy of the Genus Psoroma. J. Nat. Prod. 1989, 52, 191-192. [CrossRef]

56. Konoreva, L.; Prokopiev, I.; Frolov, I.; Chesnokov, S.; Rozhina, S.; Poryadina, L.; Shavarda, A. Metabolite profiling of the Cladonia lichens using gas chromatography-mass spectrometry. Biochem. Syst. Ecol. 2019, 85, 3-12. [CrossRef]

57. Vondrák, J.; Frolov, I.; Košnar, J.; Arup, U.; Veselská, T.; Halıc1, G.; Malíček, J.; Søchting, U. Substrate switches, phenotypic innovations and allopatric speciation formed taxonomic diversity within the lichen genus Blastenia. J. Syst. Evol. 2020, 58, 295-330. [CrossRef]

58. Frolov, I.; Vondrák, J.; Košnar, J.; Arup, U. Phylogenetic relationships within Pyrenodesmia sensu lato and the role of pigments in its taxonomic interpretation. J. Syst. Evol. 2021, 59, 454-474. [CrossRef]

59. Le Corvec, M.; Boussard-Plédel, C.; Charpentier, F.; Fatih, N.; Le Dare, B.; Massart, F.; Rojas, F.; Tariel, H.; Loréal, O.; Bureau, B.; et al. Chemotaxonomic discrimination of lichen species using an infrared chalcogenide fibre optic sensor: A useful tool for on-field biosourcing. RSC Adv. 2016, 6, 108187-108195. [CrossRef]

60. Frisvad, J.C.; Andersen, B.; Thrane, U. The use of secondary metabolite profiling in chemotaxonomy of filamentous fungi. Mycol. Res. 2008, 112, 231-240. [CrossRef]

61. Xu, M.; Heidmarsson, S.; Olafsdottir, E.S.; Buonfiglio, R.; Kogej, T.; Omarsdottir, S. Secondary metabolites from cetrarioid lichens: Chemotaxonomy, biological activities and pharmaceutical potential. Phytomedicine 2016, 23, 441-459. [CrossRef]

62. Vu, T.H.; Catheline, D.; Delmail, D.; Boustie, J.; Legrand, P.; Lohezic-Le Devehat, F. Gas chromatographic analysis to compare the fatty acid composition of fifteen lichen species, with a focus on Stereocaulon. Lichenologist 2016, 48, 323-337. [CrossRef] 